University of Warwick institutional repository: http://go.warwick.ac.uk/wrap This paper is made available online in accordance with publisher policies. Please scroll down to view the document itself. Please refer to the repository record for this item and our policy information available from the repository home page for further information.

To see the final version of this paper please visit the publisher's website. Access to the published version may require a subscription.

Author(s): V. GELFREICH and V. NAUDOT

Article Title: Analytic invariants associated with a parabolic fixed point in $\mathbb{C}^{2}$

Year of publication: 2008

Link to published version:

http://dx.doi.org/10.1017/S0143385707001046

Publisher statement: None 


\title{
Analytic invariants associated with a parabolic fixed point in $\mathbb{C}^{2}$
}

\author{
V. GELFREICH and V. NAUDOT \\ Mathematics Institute, University of Warwick, Coventry CV4 7AL, UK \\ (e-mail:V.Gelfreich@warwick.ac.uk)
}

(Received 26 March 2007 and accepted in revised form 2 November 2007)

\begin{abstract}
It is well known that in a small neighbourhood of a parabolic fixed point a real-analytic diffeomorphism of $\left(\mathbb{R}^{2}, 0\right)$ embeds in a smooth autonomous flow. In this paper we show that the complex-analytic situation is completely different and a generic diffeomorphism cannot be embedded in an analytic flow in a neighbourhood of its parabolic fixed point. We study two analytic invariants with respect to local analytic changes of coordinates. One of the invariants was introduced earlier by one of the authors. These invariants vanish for time-one maps of analytic flows. We show that one of the invariants does not vanish on an open dense subset. A complete analytic classification of the maps with a parabolic fixed point in $\mathbb{C}^{2}$ is not available at the present time.
\end{abstract}

\section{Introduction}

We consider a local diffeomorphism $F$ near a fixed point on the plane. We assume that the fixed point is at the origin, $F(0)=0$, and both eigenvalues of $F^{\prime}(0)$ are equal to 1 but $F^{\prime}(0)$ is not the identity, i.e. the origin is a parabolic fixed point of $F$. Our study of this map is motivated by the fact that under a perturbation such maps exhibit a discrete analogue of the Bogdanov-Takens bifurcation $[3,4]$. Our invariants are relevant for studying the width of a chaotic zone that appears near the bifurcation [12].

It is well known that in a small neighbourhood of a parabolic fixed point a smooth diffeomorphism of $\left(\mathbb{R}^{2}, 0\right)$ can be represented as a time-one map of a smooth autonomous flow [6]. Therefore, in the real smooth case there is no difference between local dynamics of a flow and a map. We will see that in the complex case the situation is different.

We say that $F$ formally embeds in a flow if there is a formal vector field $X$ such that $F=e^{X}$. If the series for $X$ converges, the map $F$ coincides with the time-one map of the vector field $X$ in a neighbourhood of the origin. We will see that the map $F$ formally embeds in a flow. The origin is a singular point of $X$, and the linear part of $X$ at the origin is nilpotent. 
In order to study the dynamics in a neighbourhood of a fixed point, it is convenient to make coordinate changes in order to simplify a map or a vector field as much as possible. It is well known $[\mathbf{1}, \mathbf{2}, \mathbf{5}]$ that the vector field $X$ can be transformed into the Lienard equation:

$$
\begin{aligned}
& \dot{x}=y, \\
& \dot{y}=f_{0}(x)+y f_{1}(x) .
\end{aligned}
$$

In the case of an analytic vector field this reduction can be achieved by an analytic change of variables. The Lienard equation can be further simplified using formal substitutions to eliminate infinitely many Taylor coefficients of the functions $f_{0}$ and $f_{1}$. This procedure leads to a unique formal normal form, which in general [2] still contains infinitely many terms. The coefficients of the unique formal normal form are formal invariants of $X$. Two vector fields can be formally conjugate if and only if their formal normal forms coincide. Therefore the vector field $X$ has infinitely many formal invariants.

For a given map $F$ the formal vector field $X$ is unique and, consequently, the coefficients of the unique normal form of $X$ constitute a complete set of formal invariants for the map $F$. In other words, two maps are formally conjugate if and only if the formal normal forms of the corresponding vector fields coincide.

Suppose two analytic maps are formally conjugate, is it true that they are also analytically conjugate? This question is known as the 'rigidity property'. As is usual in the case of infinitely many formal invariants, we do not know the answer to this question. Nevertheless, in this paper we construct two analytic invariants, which are independent of any finite subset of formal invariants. We conjecture that these invariants are independent of the totality of formal invariants but we are not able to prove this claim at the time of writing.

If the map $F$ preserves area, one of our two analytic invariants coincides with a splitting (Stokes) constant earlier defined in [10]. In [11] it was proved that this invariant does not vanish in the case of the area-preserving Hénon map with a parabolic fixed point, which can be written in the following form:

$$
(x, y) \mapsto\left(x+y-x^{2}, y-x^{2}\right) .
$$

This result together with analytical dependence of the invariants on parameters imply that the invariants do not vanish on an open and dense subset of maps.

The map $F$ is formally a time-one map of the formal vector field $X$, and therefore $F$ (more precisely, its Taylor series considered as a formal power series) commutes with a one-parameter family of formal maps $F_{s}=e^{s X}$ and $F=F_{1 / n}^{n}$ for every integer $n$.

If the series $X$ converges, the map $F_{S}$ is analytic in a neighbourhood of the origin. It is not difficult to check that the origin is a parabolic fixed point of $F_{S}$. Then $F_{1 / n}$ is an analytic root of $F$ with respect to composition of maps in a neighbourhood of the origin. We will show that the existence of an analytic root of some order $n>1$ implies that our analytic invariants vanish. Therefore, if $F$ is a time-one map of an analytic vector field, then its analytic invariants vanish.

We conclude that generically the map $F$ does not have any analytic root with respect to composition of maps in a neighbourhood of the origin and it does not embed in an analytic flow. 
In this paper, we prove that the analytic invariants are well defined for analytic maps with a non-degenerate parabolic fixed point, providing rigorous justification for the corresponding announcements of [12].

Our interest in this problem originates from the bifurcation theory, where many maps are naturally real-analytic. We will indicate places where real-analyticity of $F$ implies additional properties.

We note that our invariants have some similarity to holomorphic Écalle-Voronin invariants, which provided a complete classification in the one-dimensional case $[7, \mathbf{1 5}]$.

The rest of the paper is organized in the following way. In $\$ 2$ we state our main results and explain some technical details, including a rigorous definition of the analytic invariants and prove some of their properties. In $\S 3$ we construct all formal series involved in our study. Section 4 contains necessary statements from the analytic theory of linear finitedifference equations. The last two sections of the paper contain proofs of Theorems 2.1 and 2.2 respectively.

\section{Main results}

Let us describe our results in more detail. We consider a two-dimensional map $F:\left(\mathbb{C}^{2}, 0\right) \rightarrow\left(\mathbb{C}^{2}, 0\right)$ analytic in a complex neighbourhood of the origin. The origin is a parabolic fixed point of the map $F(0)=0$ and

$$
\operatorname{det} F^{\prime}(0)=1, \quad \operatorname{Tr} F^{\prime}(0)=2, \quad F^{\prime}(0) \neq \text { Id } .
$$

Equivalently, we can say that $F^{\prime}(0)$ has a double eigenvalue 1 but is not an identity matrix. Then there is a linear change of coordinates, which transforms $F^{\prime}(0)$ to the Jordan form, and we will assume that

$$
F^{\prime}(0)=\left(\begin{array}{ll}
1 & 1 \\
0 & 1
\end{array}\right) .
$$

In these coordinates $F$ takes the form $(x, y) \mapsto\left(x_{1}, y_{1}\right)$, where

$$
x_{1}=x+y+a(x, y), \quad y_{1}=y+b(x, y),
$$

where the series

$$
a(x, y)=\sum_{k+j \geq 2} a_{k j} x^{k} y^{j} \quad \text { and } \quad b(x, y)=\sum_{k+j \geq 2} b_{k j} x^{k} y^{j}
$$

converge in a neighbourhood of the origin. We say that the parabolic fixed point is nondegenerate if $b_{20} \neq 0$. In this paper we will consider only the case of a non-degenerate parabolic point. With a parabolic point we can associate a formal invariant

$$
\gamma=\frac{2 a_{20}+b_{11}}{b_{20}} .
$$

If two maps of the form (4) are formally (or analytically) conjugate, then they have the same value of this invariant. The invariant $\gamma$ is the first of an infinite sequence of formal invariants that can be associated with the map $F$. Without restricting the generality, we may assume that

$$
a_{20}=b_{20}=1 .
$$


This normalizing condition can be achieved by a substitution $(x, y) \mapsto\left(c_{1} x, c_{1} y+c_{2} x^{2}\right)$, where $c_{1}=b_{20}$ and $c_{2}=a_{20} b_{20}-b_{20}^{2}$. We will assume this normalizing condition, as it leads to a substantial simplification of the notation in the following.

Although the origin is not hyperbolic, it is well known that the map $F$ possesses stable and unstable invariant manifolds. It is convenient to represent these manifolds in a parametric form using solutions of the finite-difference equation

$$
\Gamma(\tau+1)=F(\Gamma(\tau)),
$$

which converge to 0 as $\operatorname{Re} \tau \rightarrow \pm \infty$. Respectively, '+' corresponds to the stable manifold, and '-' to the unstable one. The variable $\tau$ can be considered as an analytic coordinate on the stable or unstable invariant manifold. The restriction of $F$ onto each of the invariant manifolds is conjugate to the translation $\tau \mapsto \tau+1$.

First, we study formal solutions of equation (6) and an associated variational equation in a class of formal series that involve powers of $\tau^{-1}$ and $\log \tau$. We always assume that $\log$ stands for the main branch of the logarithm, which is positive on the positive real semiaxis. A formal solution is obtained by substituting a series into the equation, re-expanding both sides and collecting similar terms starting from the lowest order of $\tau^{-1}$. The series may diverge but each of the relations involves only a finite number of coefficients.

The formal series we get in this paper can be interpreted in two different ways. They can be considered as power series in $\tau^{-1}$ with coefficients polynomial in $\log \tau$, or as a product of a suitable factor $\tau^{k}$ and a double series in powers of two variables $\tau^{-1}$ and $\tau^{-1} \log \tau$.

We denote by $\mathbb{C}\left[\left[\tau^{-1}\right]\right]\left[\left[\tau^{-1} \log \tau\right]\right]$ the space of formal double series in powers of $\tau^{-1}$ and $\tau^{-1} \log \tau$.

Proposition 2.1. (Formal separatrix) Equation (6) has a formal solution $\hat{\boldsymbol{\Gamma}}$ of the form

$$
\hat{\boldsymbol{\Gamma}}(\tau)=\left(\begin{array}{l}
\tau^{-2} \hat{\Gamma}_{1}(\tau) \\
\tau^{-3} \hat{\Gamma}_{2}(\tau)
\end{array}\right) \quad \text { where } \hat{\Gamma}_{1}, \hat{\Gamma}_{2} \in \mathbb{C}\left[\left[\tau^{-1}\right]\right]\left[\left[\tau^{-1} \log \tau\right]\right] .
$$

Moreover, for any non-zero solution $\tilde{\boldsymbol{\Gamma}}$ from this class there is a constant $\tau_{0} \in \mathbb{C}$ such that $\tilde{\boldsymbol{\Gamma}}(\tau)=\hat{\boldsymbol{\Gamma}}\left(\tau+\tau_{0}\right)$. If additionally the map $F$ is real-analytic, the coefficients of $\hat{\boldsymbol{\Gamma}}$ can be chosen to be real.

We call the series $\hat{\boldsymbol{\Gamma}}$ a formal separatrix. Our results imply that it generically diverges. If the series converges, then it defines an analytic solution of equation (6) and represents an invariant manifold associated with the parabolic fixed point.

The formal separatrix can be written in the following coordinate form:

$$
\hat{\boldsymbol{\Gamma}}(\tau)=\left(\begin{array}{l}
\frac{6}{\tau^{2}}+\sum_{k \geq 3} \frac{x_{k}(\log \tau)}{\tau^{k}} \\
-\frac{12}{\tau^{3}}+\sum_{k \geq 4} \frac{y_{k}(\log \tau)}{\tau^{k}}
\end{array}\right),
$$

where $x_{k}$ and $y_{k}$ are polynomials of degrees $k-2$ and $k-3$ respectively. The freedom in the definition of the formal separatrix can be eliminated if we require that the polynomial $x_{3}$ does not contain a constant term. In this case it takes the form

$$
x_{3}(\log \tau)=\frac{72}{7}(\gamma-2) \log \tau \text {. }
$$


If $F$ is real-analytic, then $x_{3}$ is real on the positive real semiaxis. Moreover, analysing the proof of Proposition 2.1, we can conclude that all other terms of the formal separatrix are also real in this case.

If $\gamma=2$, then $x_{3}$ vanishes and formulae from the proof of Proposition 2.1 imply that the formal separatrix is a power series in $\tau^{-1}$ only and does not contain any logarithmic term at all, i.e. all $x_{k}$ and $y_{k}$ are constant. In particular, this condition is satisfied if $F$ is reversible or area-preserving. The area-preserving and reversible cases are covered by our general theory.

We say that $\hat{U}$ is a formal fundamental solution of the variational equation if $\hat{U}$ is a $2 \times 2$ matrix-valued formal series that satisfies the equation

$$
\hat{\mathrm{U}}(\tau+1)=F^{\prime}(\hat{\Gamma}(\tau)) \hat{\mathrm{U}}(\tau)
$$

and two normalizing conditions:

(1) the constant term of the series $\hat{w}_{0}(\tau):=\operatorname{det} \hat{U}(\tau)$ is equal to 1 ; and

(2) the second column of $\hat{U}(\tau)$ coincides with $\hat{\boldsymbol{\Gamma}}^{\prime}(\tau)$.

Note that the last condition is quite natural as differentiating equation (6) we conclude that $\hat{\boldsymbol{\Gamma}}^{\prime}$ satisfies the variational equation:

$$
\hat{\boldsymbol{\Gamma}}^{\prime}(\tau+1)=F^{\prime}(\hat{\boldsymbol{\Gamma}}(\tau)) \hat{\boldsymbol{\Gamma}}^{\prime}(\tau)
$$

We define an auxiliary matrix

$$
J_{d}=\left(\begin{array}{ll}
1 & 0 \\
d & 1
\end{array}\right)
$$

PROPOSITION 2.2. (Formal fundamental solution) Equation (9) has a formal fundamental solution $\hat{\mathrm{U}}$ of the form

$$
\hat{\mathrm{U}}(\tau)=\left(\begin{array}{ll}
\tau^{4} \hat{U}_{11}(\tau) & \tau^{-3} \hat{U}_{12}(\tau) \\
\tau^{3} \hat{U}_{11}(\tau) & \tau^{-4} \hat{U}_{12}(\tau)
\end{array}\right) \quad \text { where } \hat{U}_{k l} \in \mathbb{C}\left[\left[\tau^{-1}\right]\right]\left[\left[\tau^{-1} \log \tau\right]\right],
$$

which satisfies both normalizing conditions. If $\tilde{\mathrm{U}}$ is another normalized formal solution of equation (9), then there is a constant $d \in \mathbb{C}$ such that

$$
\tilde{\mathrm{U}}(\tau)=\hat{\mathrm{U}}(\tau) J_{d} \text {. }
$$

Moreover, if the map $F$ is real-analytic and the coefficients of $\hat{\Gamma}$ are chosen to be real, then the coefficients of $\hat{\mathrm{U}}$ can be chosen to be real.

Now we consider analytical solutions of equation (6). Let us fix a positive number $\delta_{0} \in(0, \pi / 2)$ such that $\tan \delta_{0}<1 / 2$. This value will be the same in all our statements and we omit the dependence on that parameter to shorten the notation. For $r>0$ consider the sector (see Figure 1):

$$
D_{+}(r)=\left\{\tau \in \mathbb{C}|| \arg (x-r) \mid<\pi-\delta_{0}\right\} .
$$

THEOREM 2.1. (Analytic stable manifold) Let $F$ be a local analytic diffeomorphism with a non-degenerate parabolic fixed point at the origin and $\hat{\boldsymbol{\Gamma}}$ be its formal separatrix. Then there are a constant $r_{0}>0$ and a unique analytic function $\Gamma^{s}: D_{+}\left(r_{0}\right) \rightarrow \mathbb{C}^{2}$, which satisfies (6) and is asymptotic to $\hat{\boldsymbol{\Gamma}}(\tau)$ as $\tau \rightarrow \infty, \tau \in D_{+}\left(r_{0}\right)$. Moreover, if $F$ is realanalytic and the coefficients of $\hat{\boldsymbol{\Gamma}}$ are chosen to be real, then $\boldsymbol{\Gamma}^{s}$ is also real-analytic. 

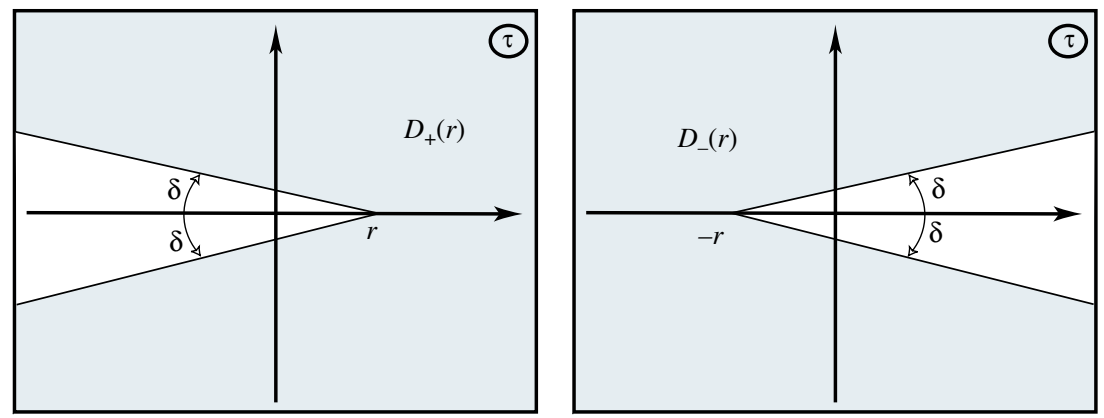

FIGURE 1. Domains $D_{+}(r)$ and $D_{-}(r)$.

We use the following notation:

$$
\Gamma^{s}(\tau) \simeq \hat{\boldsymbol{\Gamma}}(\tau) \quad \text { as } \tau \rightarrow \infty, \quad \tau \in D_{+}\left(r_{0}\right),
$$

means that the function $\Gamma^{s}$ is asymptotic to the series $\hat{\Gamma}$, i.e. the difference between the function and a partial sum of the series is of the same order as the first omitted term.

If a point belongs to $\Gamma^{s}\left(D_{+}\left(r_{0}\right)\right)$, its forward iterates converge to the origin. Therefore $\Gamma^{s}$ represents the local stable manifold of the parabolic fixed point. We think that any point that is attracted by the origin eventually belongs to this local stable manifold. The proof of this fact is not known at the time of writing.

In order to study the unstable manifold we note that the unstable manifold is a stable manifold of the inverse map. We consider a symmetric domain $D_{-}(r)=-D_{+}(r)$ (see Figure 1). The logarithm has two analytic continuations from $D_{+}(r)$ onto $D_{-}(r)$ obtained by clockwise and anticlockwise analytic continuations. The difference between these two branches of $\log$ equals $2 \pi i$. Then the formal series $\hat{\boldsymbol{\Gamma}}$ can be interpreted in two different ways $\hat{\boldsymbol{\Gamma}}_{+}$and $\hat{\boldsymbol{\Gamma}}_{-}$depending on the choice of the branch of log. Proposition 2.1 implies that there is $\tau_{0} \in \mathbb{C}$ such that $\hat{\boldsymbol{\Gamma}}_{+}(\tau)=\hat{\boldsymbol{\Gamma}}_{-}\left(\tau-\tau_{0}\right)$. We can find this constant explicitly. Indeed, taking into account that $\hat{\Gamma}$ can be fixed by the condition (8), we obtain

$$
x_{3,+}(\tau)-x_{3,-}(\tau)=\frac{144 \pi i}{7}(\gamma-2),
$$

independently of the normalization of $\hat{\boldsymbol{\Gamma}}$. Taking into account the first two orders of the first line of (7) and re-expanding $\hat{\boldsymbol{\Gamma}}_{-}\left(\tau-\tau_{0}\right)$, we conclude that

$$
\tau_{0}=\frac{12 \pi i}{7}(\gamma-2) \text {. }
$$

Applying Theorem 2.1 to $F^{-1}$, we obtain the following two statements.

COROLLARY 2.1. (Analytic unstable manifold) There is a constant $r_{0}^{\prime}>0$ such that:

(1) there is a unique solution $\boldsymbol{\Gamma}=\boldsymbol{\Gamma}_{-}^{u}$ of equation (6), which is analytic in $D_{-}\left(r_{0}^{\prime}\right)$ and $\boldsymbol{\Gamma}_{-}^{u}(\tau) \simeq \hat{\boldsymbol{\Gamma}}_{-}(\tau)$ as $\tau \rightarrow \infty$ in $D_{-}\left(r_{0}^{\prime}\right)$; and

(2) there is a unique solution $\Gamma=\Gamma_{+}^{u}$ of equation (6), which is analytic in $D_{-}\left(r_{0}^{\prime}\right)$ and $\boldsymbol{\Gamma}_{+}^{u}(\tau) \simeq \hat{\boldsymbol{\Gamma}}_{+}(\tau)$ as $\tau \rightarrow \infty$ in $D_{-}\left(r_{0}^{\prime}\right)$. 
Uniqueness of the analytic solutions implies that $\boldsymbol{\Gamma}_{+}^{u}(\tau)=\boldsymbol{\Gamma}_{-}^{u}\left(\tau-\tau_{0}\right)$. Therefore, these two solutions represent two different parameterizations of the unique local unstable manifold. If $\gamma \neq 2$, none of the series $\hat{\boldsymbol{\Gamma}}_{ \pm}$is real on the negative real semiaxis even in the case of a real-analytic $F$. Nevertheless, Theorem 2.1 applied to $F^{-1}$ also implies the existence of a real-analytic parameterization of the unstable manifold in the realanalytic case.

The solutions $\Gamma_{-}^{u}$ and $\Gamma^{s}$ have a common asymptotic expansion in the lower component of $D_{+}\left(r_{0}\right) \cap D_{-}\left(r_{0}\right)$, which implies that their difference decreases faster than any power of $\tau^{-1}$. We prove a more delicate estimate on this difference. Let $r_{1}=\max \left\{r_{0}, r_{0}^{\prime}\right\}$ and consider two domains:

$$
D_{1}^{ \pm}\left(r_{1}\right)=D_{+}\left(r_{1}\right) \cap D_{-}\left(r_{1}\right) \cap\{ \pm \operatorname{Im} \tau>0\} .
$$

THEOREM 2.2. (Exponential asymptotic for the separatrix splitting) There are two vectors $\Theta^{-}, \Theta^{+} \in \mathbb{C}^{2}$ such that

$$
\begin{aligned}
& \Gamma_{-}^{u}(\tau)-\Gamma^{s}(\tau) \simeq e^{-2 \pi i \tau} \hat{\mathrm{U}}(\tau) \Theta^{-} \quad \text { as } \tau \rightarrow \infty, \tau \in D_{1}^{-}\left(r_{1}\right), \\
& \Gamma_{+}^{u}(\tau)-\Gamma^{s}(\tau) \simeq e^{2 \pi i \tau} \hat{\mathrm{U}}(\tau) \Theta^{+} \quad \text { as } \tau \rightarrow \infty, \tau \in D_{1}^{+}\left(r_{1}\right) .
\end{aligned}
$$

Moreover, if additionally $F$ is real-analytic and all coefficients of the series $\hat{\boldsymbol{\Gamma}}$ and $\hat{\mathrm{U}}$ are real, then $\Theta^{+}=\bar{\Theta}^{-}$.

Note that this theorem implies an exponentially small upper bound for the splitting of invariant manifolds of the map $F$. Namely, let us write the parameterizations in the coordinate form:

$$
\Gamma^{s}(\tau)=\left(x^{s}(\tau), y^{s}(\tau)\right) \quad \text { and } \quad \Gamma_{-}^{u}(\tau)=\left(x_{-}^{u}(\tau), y_{-}^{u}(\tau)\right) .
$$

Then (12) and (11) imply that there is a constant $K>0$ such that

$$
\begin{aligned}
& \left|x_{-}^{u}(\tau)-x^{s}(\tau)\right|<K|\tau|^{4} e^{2 \pi \operatorname{Im} \tau}, \\
& \left|y_{-}^{u}(\tau)-y^{s}(\tau)\right|<K|\tau|^{3} e^{2 \pi \operatorname{Im} \tau},
\end{aligned}
$$

for all $\tau \in D_{1}^{-}\left(r_{1}\right)$. A similar upper bound is valid in $D_{1}^{+}\left(r_{1}\right)$. Write $\Theta^{ \pm}=$ $\left(\Theta_{1}^{ \pm}, \Theta_{2}^{ \pm}\right)$. If $\Theta_{1}^{ \pm} \neq 0$, the theorem also implies a lower bound for the splitting distance $\left\|\boldsymbol{\Gamma}_{ \pm}^{u}(\tau)-\Gamma^{s}(\tau)\right\|$.

The constants $\Theta^{ \pm}$are not uniquely defined due to the freedom in the choice of the formal series $\hat{\Gamma}$ and $\hat{U}$, which also causes the non-uniqueness of analytic parameterizations for the invariant manifolds. This freedom can be eliminated by setting conditions on terms of orders $\tau^{-3}$ and $\tau^{-4}$. Unfortunately, these additional conditions cannot be restated in terms of formal invariants of the map $F$. Therefore, the freedom in the definition of $\Theta^{ \pm}$ cannot be eliminated in a coordinate-independent way.

Indeed, suppose we found a rule that eliminates the freedom in the definition of the constant term of $x_{3}$, and depends only on the coefficients of the Taylor series for $F$ up to order $n$. Recall that $\Theta^{ \pm}=\left(\Theta_{1}^{ \pm}, \Theta_{2}^{ \pm}\right)$. Values of $\Theta_{1}^{ \pm}$do not depend on the freedom in the definition of the formal series $\hat{U}(\tau)$. Therefore, this rule eliminates the freedom in the definition of $\Theta_{1}^{ \pm}$. 
For any map $F$ and any natural number $n$ we construct a map $F_{n}$, which is analytically conjugate to $F$ and has the same $n$-jet. Moreover, the application of the rule to $F$ and $F_{n}$ leads to different values of the constants $\Theta_{1}^{ \pm}$.

Later we will prove that there is a formal vector field $X$ such that $F=e^{X}$. It follows that $F$ commutes with the formal series $e^{s X}$ for every $s \in \mathbb{C}$. Let $H_{n}=e^{s[X]_{n}}$, where $[X]_{n}$ denotes the partial sum of the series $X$ up to an order $n$. Then the Taylor series of the map

$$
\tilde{F}_{n}=H_{n} \circ F \circ H_{n}^{-1}
$$

coincides with the Taylor series of $F$ up to the order $n$. Let $\hat{\boldsymbol{\Gamma}}$ and $\hat{\boldsymbol{\Gamma}}_{n}$ be formal separatrices of the maps $F$ and $\tilde{F}_{n}$ respectively. The rule implies that the coefficients of order $\tau^{-3}$ of those two formal series coincide.

On the other hand, the equation

$$
\hat{\boldsymbol{\Gamma}}_{n}(\tau+1)=\tilde{F}_{n} \circ \hat{\boldsymbol{\Gamma}}_{n}(\tau)
$$

has an obvious solution $H_{n} \circ \hat{\boldsymbol{\Gamma}}(\tau)$. Comparing the terms of order $\tau^{-3}$ in the first component, we conclude by uniqueness that

$$
\hat{\boldsymbol{\Gamma}}_{n}(\tau)=H_{n} \circ \hat{\boldsymbol{\Gamma}}(\tau-s) .
$$

There is a formal fundamental solution $\hat{\mathrm{U}}_{n}(\tau)=H_{n}^{\prime}(\hat{\boldsymbol{\Gamma}}(\tau-s)) \tilde{\mathrm{U}}(\tau-s)$. Then using the definition of $\Theta^{ \pm}$we obtain

$$
\tilde{\Theta}^{-}=e^{2 \pi i s} \Theta^{-} \text {and } \tilde{\Theta}^{+}=e^{-2 \pi i s} \Theta^{+} .
$$

Therefore $\tilde{\Theta}^{+} \neq \Theta^{+}$if $s \notin \mathbb{Z}$.

We have constructed two analytically conjugated maps $F$ and $\tilde{F}_{n}$, with different values of $\Theta_{1}^{ \pm}$.

The following theorem shows that we can construct two analytic invariants of the map $F$ from four constants $\Theta_{j}^{ \pm}, j=1,2$.

THEOREM 2.3. (On analytic invariants) The two complex constants

$$
\Phi_{1}=\Theta_{1}^{-} \Theta_{1}^{+} \text {and } \Phi_{2}=\operatorname{det}\left(\begin{array}{cc}
\Theta_{1}^{-} & \Theta_{1}^{+} \\
\Theta_{2}^{-} & \Theta_{2}^{+}
\end{array}\right)
$$

are independent of the freedom in the choice of the formal series $\hat{\Gamma}$ and $\hat{\mathrm{U}}$, which are involved in the definition of $\Theta_{j}^{ \pm}, j=1,2$. Moreover, $\Phi_{1}$ and $\Phi_{2}$ are invariant under analytic conjugacy.

In the case of a real-analytic $F$, the $\hat{\boldsymbol{\Gamma}}$ and $\hat{U}$ can be chosen to be real-analytic. Then $\Theta^{-}=\bar{\Theta}^{+}$and the analytic invariants are real:

$$
\Phi_{1}=\Theta_{1}^{-} \Theta_{1}^{+}=\left|\Theta_{1}^{-}\right|^{2} \text { and } \Phi_{2}=\operatorname{det}\left(\begin{array}{cc}
\Theta_{1}^{-} & \Theta_{1}^{+} \\
\Theta_{2}^{-} & \Theta_{2}^{+}
\end{array}\right)=2 \operatorname{Im}\left(\Theta_{1}^{-} \bar{\Theta}_{2}^{-}\right) .
$$

Proof of Theorem 2.3. First we check that the analytic invariants are independent of the freedom in the choice of the formal expansions. Let $\hat{\boldsymbol{\Gamma}}$ and $\hat{\tilde{\Gamma}}$ be two formal separatrices, and $\hat{\mathrm{U}}$ and $\hat{\tilde{U}}$ be two formal basic solutions. Then there are $\tau_{0}, d \in \mathbb{C}$ such that

$$
\hat{\tilde{\boldsymbol{\Gamma}}}(\tau)=\hat{\boldsymbol{\Gamma}}\left(\tau-\tau_{0}\right), \quad \hat{\tilde{\mathrm{U}}}(\tau)=\hat{\mathrm{U}}\left(\tau-\tau_{0}\right) J_{d},
$$


where $J_{d}$ is defined by (10). Then

$$
\tilde{\boldsymbol{\Gamma}}^{s}(\tau)=\boldsymbol{\Gamma}^{s}\left(\tau-\tau_{0}\right) \quad \text { and } \quad \tilde{\boldsymbol{\Gamma}}_{ \pm}^{u}(\tau)=\boldsymbol{\Gamma}_{ \pm}^{u}\left(\tau-\tau_{0}\right) .
$$

Therefore

$$
\tilde{\boldsymbol{\Gamma}}_{ \pm}^{u}(\tau)-\tilde{\boldsymbol{\Gamma}}^{s}(\tau)=\boldsymbol{\Gamma}_{ \pm}^{u}\left(\tau-\tau_{0}\right)-\boldsymbol{\Gamma}^{s}\left(\tau-\tau_{0}\right) \simeq e^{\mp 2 \pi i\left(\tau-\tau_{0}\right)} \hat{\mathrm{U}}\left(\tau-\tau_{0}\right) \Theta^{ \pm} .
$$

On the other hand

$$
\tilde{\boldsymbol{\Gamma}}_{ \pm}^{u}(\tau)-\tilde{\boldsymbol{\Gamma}}^{s}(\tau) \simeq e^{\mp 2 \pi i \tau} \hat{\tilde{\mathrm{U}}}(\tau) \tilde{\Theta}^{ \pm}=e^{\mp 2 \pi i \tau} \hat{\mathrm{U}}\left(\tau-\tau_{0}\right) J_{d} \tilde{\Theta}^{ \pm} .
$$

Comparing these two asymptotic expansions, we conclude that

$$
e^{ \pm 2 \pi i \tau_{0}} \Theta^{ \pm}=J_{d} \tilde{\Theta}^{ \pm}
$$

Taking into account the triangular form of the matrix $J_{d}$, we see that $\Theta_{1}^{+} \Theta_{1}^{-}=\tilde{\Theta}_{1}^{+} \tilde{\Theta}_{1}^{-}$. Moreover, det $J_{d}=1$ implies that $\operatorname{det}\left(\Theta^{-}, \Theta^{+}\right)=\operatorname{det}\left(\tilde{\Theta}^{-}, \tilde{\Theta}^{+}\right)$. Therefore, the invariants are independent of the choice of the formal series.

Now let us prove that $\Phi_{1}$ and $\Phi_{2}$ are invariant under analytic conjugacy. Let $H$ be a local analytic diffeomorphism in a neighbourhood of the origin. Consider the map

$$
\tilde{F}=H \circ F \circ H^{-1},
$$

and assume that $\tilde{F}$ is also of the form (4) with the series $a, b$ replaced by $\tilde{a}, \tilde{b}$. Then necessarily there is $c \in \mathbb{C}$ such that

$$
H^{\prime}(0)=\left(\begin{array}{ll}
1 & c \\
0 & 1
\end{array}\right)
$$

A formal separatrix and a formal fundamental solution for $\tilde{F}$ can be defined by

$$
\hat{\tilde{\boldsymbol{\Gamma}}}=H \circ \hat{\boldsymbol{\Gamma}}, \quad \hat{\tilde{U}}=\left(H^{\prime} \circ \hat{\boldsymbol{\Gamma}}\right) \hat{\mathrm{U}} .
$$

Then

$$
\tilde{\boldsymbol{\Gamma}}_{ \pm}^{u}(\tau)-\tilde{\boldsymbol{\Gamma}}^{s}(\tau)=H\left(\boldsymbol{\Gamma}^{s}(\tau)\right)-H\left(\boldsymbol{\Gamma}_{ \pm}^{u}(\tau)\right) \simeq H^{\prime}(\hat{\boldsymbol{\Gamma}}(\tau)) e^{\mp 2 \pi i \tau} \hat{\mathrm{U}}(\tau) \tilde{\Theta}^{ \pm}
$$

On the other hand

$$
\tilde{\boldsymbol{\Gamma}}_{ \pm}^{u}(\tau)-\tilde{\boldsymbol{\Gamma}}^{s}(\tau) \simeq e^{\mp 2 \pi i \tau} \hat{\tilde{\mathrm{U}}}(\tau) \tilde{\Theta}^{ \pm}=e^{\mp 2 \pi i \tau} H^{\prime}(\hat{\boldsymbol{\Gamma}}(\tau)) \hat{\mathrm{U}}(\tau) \tilde{\Theta}^{ \pm}
$$

Therefore this choice of $\hat{\tilde{\Gamma}}$ and $\hat{\tilde{U}}$ leads to

$$
\tilde{\Theta}^{ \pm}=\Theta^{ \pm} .
$$

By the first part of the theorem, any other choice leads to the same values of the invariants $\Phi_{k}, k=1,2$.

THEOREM 2.4. (Analytic dependence on a parameter) If $F_{v}$ is an analytic family of maps that for every fixed $v$ satisfy the assumptions of the previous theorems, then there is a choice of formal solutions $\hat{\boldsymbol{\Gamma}}$ and $\hat{\mathrm{U}}$ such that $\Theta=\Theta_{v}$ are analytic functions of $v$. 
This theorem implies that $\Phi_{j}$ are analytic in $v$ because they do not depend on the choice of the formal series.

The coefficients of formal expansions are polynomial in Taylor coefficients of the map $F_{v}$ and consequently are analytic in $v$ provided the free coefficients of asymptotic expansions are chosen analytic in $\nu$. In order to show the analyticity of $\Theta_{\nu}$, it remains to trace the proofs of their existence for a fixed $v$ and check the uniformity of the estimates involved.

It was proved in [11] that in the case of the Hénon map the first component of $\Theta$ does not vanish.

Corollary 2.2. One has $\Theta_{1}^{+} \Theta_{1}^{-} \neq 0$ for a generic $F$.

COROLlary 2.3. A generic $F$ cannot be embedded in an analytical flow.

In order to derive the last statement, we check that, if $X$ is an analytic vector field with a non-degenerate parabolic fixed point and $F_{S}$ is its time-s map, then $F_{S}$ satisfies the assumptions of Theorem 2.2 for any fixed $s \neq 0$ and the corresponding constants $\Theta_{s}^{ \pm}$vanish.

It is sufficient to consider two values: $s=1 / 2$ and $s=1$. Obviously, $F_{1}=F_{1 / 2} \circ F_{1 / 2}$. We check that, if $\Gamma^{u, s}(\tau ; s)$ is an additive parameterization of the stable and unstable manifolds for $F_{s}$, then

$$
\Gamma^{u, s}\left(\tau+2 ; \frac{1}{2}\right)=F_{1 / 2}^{2}\left(\Gamma^{u, s}\left(\tau ; \frac{1}{2}\right)\right)=F_{1}\left(\Gamma^{u, s}\left(\tau ; \frac{1}{2}\right)\right) .
$$

We conclude that

$$
\Gamma^{u, s}(\tau ; 1)=\Gamma^{u, s}\left(2 \tau ; \frac{1}{2}\right)
$$

satisfies equation (6). The exponential asymptotic (12) for $F_{1 / 2}$ implies that

$$
\Gamma_{ \pm}^{u}(\tau ; 1)-\Gamma^{u, s}(\tau ; 1)=\Gamma_{ \pm}^{u}\left(2 \tau ; \frac{1}{2}\right)-\Gamma^{u, s}\left(2 \tau ; \frac{1}{2}\right) \asymp e^{ \pm 4 \pi i \tau} \hat{\mathrm{U}}_{1 / 2}(2 \tau) \Theta_{1 / 2}^{ \pm} .
$$

On the other hand, Theorem 2.2 can be applied directly to $F_{1}$ and implies that

$$
\Gamma_{ \pm}^{u}(\tau ; 1)-\Gamma^{u, s}(\tau ; 1) \asymp e^{ \pm 2 \pi i \tau} \hat{\mathrm{U}}_{1}(\tau) \Theta_{1}^{ \pm} .
$$

Comparing these two asymptotic expansions and taking into account that $\hat{\mathrm{U}}$ grows no faster than $O\left(\tau^{4}\right)$, we conclude that $\Theta_{1}^{ \pm}=0$.

\section{Formal expansions}

3.1. Formal embedding into a flow. In this subsection we show that the map $F$ can be formally considered as a time-one map of a vector field $X$. The vector field $X$ is constructed in the form of a power series. Although all the coefficients of the series are uniquely defined, there is no reason to expect convergence of the series for a generic $F$. Actually, the divergence of the series for an open dense set follows from the fact that the analytic invariant for a time-one map of a flow vanishes and we prove that for a generic $F$ the invariant is different from zero.

Proposition 3.1. Let $F$ be analytic in a neighbourhood of the origin,

$$
F(0)=0 \quad \text { and } \quad F^{\prime}(0)=\left(\begin{array}{ll}
1 & 1 \\
0 & 1
\end{array}\right) .
$$

Then there is a unique formal vector field $X$ such that $F(u, v)=e^{X}(u, v)$. 
Proof. It is convenient to introduce a calibrating function on the space of formal power series in $u$ and $v$. Namely, we let $\delta\left(u^{m} v^{n}\right)=2 m+3 n$. We say that $u^{m} v^{n}$ is a $\delta$ homogeneous monomial of the order $\delta\left(u^{m} v^{n}\right)$.

Let us expand $a(u, v)$ and $b(u, v)$ into Taylor series. We slightly overload our notation by keeping the same letter for an analytic function and its Taylor series.

We represent the formal vector field $X$ as a sum of $\delta$-homogeneous polynomial vector fields

$$
X=\sum_{k \geq 1} X_{k}, \quad X_{k}=p_{k+3}(u, v) \partial_{u}+q_{k+4}(u, v) \partial_{v},
$$

where $p_{k}$ and $q_{k}$ are $\delta$-homogeneous polynomials of $\delta$-order $k$. We note that when we apply $X_{k}$ to a $\delta$-homogeneous polynomial of $\delta$-order $n$ we obtain a $\delta$-homogeneous polynomial of $\delta$-order $n+k$.

Now let $\pi_{k}$ denote a projection of a formal power series onto the subspace of $\delta$ homogeneous polynomials of $\delta$-order $k$.

The equation $F(u, v)=e^{X}(u, v)$ can be written in the coordinate form:

$$
\begin{gathered}
u+v+a(u, v)=e^{X} u, \\
v+b(u, v)=e^{X} v .
\end{gathered}
$$

Here

$$
a(u, v)=\sum_{k+l \geq 2} a_{k l} u^{k} v^{l} \quad \text { and } \quad b(u, v)=\sum_{k+l \geq 2} b_{k l} u^{k} v^{l} .
$$

Let us consider its projections onto $\delta$-homogeneous polynomials. Taking into account the definition of the exponent, $e^{X}=\mathrm{id}+\sum_{n \geq 1}(1 / n !) X^{n}$, and the equalities

$$
X u=\sum_{k \geq 4} p_{k}(u, v) \quad \text { and } \quad X v=\sum_{k \geq 5} q_{k}(u, v),
$$

and considering the leading orders, we obtain

$$
X_{1}=v \partial_{u}+b_{20} u^{2} \partial_{v} .
$$

The other polynomials $p_{k}$ and $q_{k}$ are recursively defined by the formulae:

$$
\begin{aligned}
p_{k} & =\pi_{k}\left(a(u, v)-\left(e^{X}-1-X\right) u\right), \\
q_{k+1} & =\pi_{k+1}\left(b(u, v)-\left(e^{X}-1-X\right) v\right), \quad k \geq 2 .
\end{aligned}
$$

It is easy to see that the right-hand sides of these equalities are finite sums, which depend on $p_{m}$ with $2 \leq m \leq k-1$ and $q_{n}$ with $3 \leq n \leq k-2$ as well as on the Taylor coefficients of the functions $a$ and $b$. Therefore, these polynomials are uniquely defined.

3.2. Formal series for the invariant manifolds. In this section we provide the proof of Proposition 2.1. Namely, we construct the formal series in powers of $\tau^{-1}$ and $\tau^{-1} \log \tau$, which represent the 'formal' invariant manifold associated with the parabolic fixed point of $F$. If the series were convergent, its sum would be an analytic function, and its image would be an invariant manifold of $F$. In general, we do not expect the series to converge. 
The proposition can be proved by substituting the formal series

$$
\hat{\boldsymbol{\Gamma}}(\tau)=\left(\begin{array}{l}
\sum_{k \geq 2} \frac{x_{k}(\log \tau)}{\tau^{k}} \\
\sum_{k \geq 3} \frac{y_{k}(\log \tau)}{\tau^{k}}
\end{array}\right),
$$

into equation (6), re-expanding both sides of the equation, and finally collecting the terms of the same order in $\tau$. This leads to a recurrent system on the coefficients $x_{k}$ and $y_{k}$. In this way we get

$$
x_{2}=6, \quad y_{3}=-12, \quad x_{3}=x_{31} \log \tau+x_{30}, \quad y_{4}=-3 x_{41} \log \tau+y_{40},
$$

where

$$
x_{31}=\frac{72}{7}(\gamma-2), \quad y_{40}=\frac{18}{7}(4 \gamma-15)-3 x_{30} .
$$

We also used the normalizing condition (5) to simplify the expressions for the coefficients. The constant $x_{30}$ remains free. All other coefficients at all orders are determined uniquely after $x_{30}$ is fixed. This provides the desired formal separatrix.

An alternative proof can be based on the interpolating vector field described in the previous subsection. We sketch the corresponding arguments.

Let $X$ be the interpolating vector field, which exists by Proposition 3.1. Any formal solution of the equation

$$
\dot{\boldsymbol{\Gamma}}_{0}=X \circ \boldsymbol{\Gamma}_{0},
$$

simultaneously satisfies equation (6) and vice versa.

There is a formal close-to-identity change of coordinates, which conjugates $X$ to the Lienard equation (1). It is not difficult to check that

$$
f_{0}(x)=x^{2}+O\left(x^{3}\right) \text { and } f_{1}(x)=(\gamma-2) x+O\left(x^{2}\right) .
$$

Now we look for a formal solution of the Lienard equation in the form

$$
x(\tau)=\sum_{n \geq 2} \frac{1}{\tau^{n}} \sum_{k=0}^{n-2} p_{n, k} \log ^{k} \tau .
$$

We substitute the series into the differential equation and collect the terms of order $\tau^{-4}$ and $\tau^{-5}$. As a result we see that

$$
p_{2,0}=6, \quad p_{3,1}=\frac{72}{7}(\gamma-2),
$$

and there is no condition on $p_{3,0}$. We continue by induction to check that collecting the terms of order $t^{n+2} \log ^{k} t$ we obtain an equation of the form

$$
n(n+1) p_{n, k}=2 p_{2,0} p_{n, k}+\varphi_{n, k},
$$

where $\varphi_{n, k}$ is a polynomial function that depends on $p_{m, j}$ with $m<n$ and $p_{n, j}$ with $k<j \leq n-2$. Therefore we obtain an infinite triangular system, which has a unique solution for every fixed $p_{3,0}$.

We obtain the formal solution $\hat{\boldsymbol{\Gamma}}$ by applying the inverse change of coordinates to the formal vector-valued series $(x(\tau), \dot{x}(\tau))$ obtained from the formal solution of the Lienard equation. This completes the proof. 
We note that the formal solution has the following important property. If we denote by $[\hat{\boldsymbol{\Gamma}}]_{n}(t)$ the sum of the first $n$ terms of the formal series $\hat{\boldsymbol{\Gamma}}$, then the $x$ and $y$ components of the residue term

$$
R_{n}(\tau)=[\hat{\boldsymbol{\Gamma}}]_{n}(\tau+1)-F\left([\hat{\boldsymbol{\Gamma}}]_{n}(\tau)\right)
$$

can be bounded from above by $O\left(\log ^{n+1} \tau^{n+3}\right)$ and $O\left(\log ^{n+1} \tau^{n+4}\right)$, respectively.

3.3. Formal fundamental solutions. In this section we study a homogeneous linear equation of the form

$$
\hat{\mathrm{U}}(\tau+1)=\hat{\mathrm{A}}(\tau) \hat{\mathrm{U}}(\tau) .
$$

The proof is a combinatorial study of the equations obtained by a formal substitution of the series and by collecting terms of the same order in $\tau^{-1}$.

LEMMA 3.1. Suppose that

$$
\hat{\mathrm{A}}(\tau)=\left(\begin{array}{ll}
1 & 1 \\
0 & 1
\end{array}\right)+\left(\begin{array}{ll}
\hat{A}_{11}(\tau) & \hat{A}_{12}(\tau) \\
\hat{A}_{21}(\tau) & \hat{A}_{22}(\tau)
\end{array}\right),
$$

where $\hat{A}_{11}(\tau), \hat{A}_{12}(\tau), \hat{A}_{21}(\tau)$ and $\hat{A}_{22}(\tau)$ are formal series from the class $\tau^{-2} \mathbb{C}\left[\left[\tau^{-1}\right]\right]\left[\left[\tau^{-1} \log \tau\right]\right]$ with a fixed leading term $A_{21,-2}=12$. Then the equation

$$
\hat{\boldsymbol{\Phi}}(\tau+1)=\hat{\mathrm{A}}(\tau) \hat{\boldsymbol{\Phi}}(\tau)
$$

admits two formal solutions $\hat{\boldsymbol{\Phi}}^{+}=\left(\hat{\phi}^{+}, \hat{\psi}^{+}\right)$and $\hat{\boldsymbol{\Phi}}^{-}=\left(\hat{\phi}^{-}, \hat{\psi}^{-}\right)$, where

$$
\left\{\begin{array}{l}
\hat{\phi}^{+}(\tau)=\sum_{k \leq 4} \phi_{k}^{+}(\log \tau) \tau^{k} \\
\hat{\psi}^{+}(\tau)=\sum_{k \leq 3} \psi_{k}^{+}(\log \tau) \tau^{k}
\end{array}\right.
$$

and

$$
\left\{\begin{array}{l}
\hat{\phi}^{-}(\tau)=\sum_{k \leq-3} \phi_{k}^{-}(\log \tau) \tau^{k}, \\
\hat{\psi}^{-}(\tau)=\sum_{k \leq-4} \psi_{k}^{-}(\log \tau) \tau^{k}
\end{array}\right.
$$

Moreover, $\phi_{k}^{+}$and $\psi_{k-1}^{+}$are polynomials of degree not greater than $4-k$ when $-3<$ $k \leq 4$ and of degree not greater than $5-k$ when $k \leq-3$, both $\phi_{k}^{-}$and $\psi_{k-1}^{-}$being of degree $-3-k$.

Proof. We prove the lemma for $\hat{\boldsymbol{\Phi}}^{+}$, the proof for $\hat{\boldsymbol{\Phi}}^{-}$being completely similar. We therefore remove the exponent ' + ' from now on since there is no confusion. The proof is done by induction on $n$. We first write

$$
\hat{\boldsymbol{\Phi}}(\tau)=\sum_{k \leq 4} \boldsymbol{\Phi}_{k}(\tau, \log \tau),
$$

where

$$
\boldsymbol{\Phi}_{k}(\tau, \log \tau)=\left(\tau^{k} \phi_{k}(\log \tau), \tau^{k-1} \psi_{k-1}(\log \tau)\right) .
$$


In the class of formal series $\hat{\boldsymbol{\Phi}}(\tau+1)=\exp (\partial / \partial \tau) \hat{\boldsymbol{\Phi}}(\tau)$, which is equivalent to

$$
\hat{\boldsymbol{\Phi}}(\tau+1)=\sum_{i \geq 0} \frac{(\hat{\boldsymbol{\Phi}})^{(i)}(\tau)}{i !} .
$$

Moving the first term of the sum to the left-hand side and substituting (20) we obtain

$$
\hat{\boldsymbol{\Phi}}(\tau+1)-\hat{\boldsymbol{\Phi}}(\tau)=\sum_{i \geq 1} \frac{1}{i !} \sum_{k \leq 4} \frac{d^{i}}{d \tau^{i}}\left(\boldsymbol{\Phi}_{k}(\tau, \log \tau)\right) .
$$

It is convenient to re-order the terms of the sum to group together terms of the same order in $\tau$ :

$$
\hat{\boldsymbol{\Phi}}(\tau+1)-\hat{\boldsymbol{\Phi}}(\tau)=\sum_{n \leq 3} \sum_{l=n+1}^{4} \frac{1}{(l-n) !} \frac{d^{l-n}}{d \tau^{l-n}}\left(\Phi_{l}(\tau, \log \tau)\right) .
$$

Equation (17) can be written in the form

$$
\boldsymbol{\Phi}(\tau+1)-\boldsymbol{\Phi}(\tau)=(\hat{\mathrm{A}}(\tau)-\mathrm{Id}) \boldsymbol{\Phi}(\tau) .
$$

Substituting (16) and (23) into the equation, we see that

$$
\begin{aligned}
& \sum_{n \leq 3} \sum_{l=n+1}^{4} \frac{1}{(l-n) !} \frac{d^{l-n}}{d \tau^{l-n}}\left(\phi_{l}(\log \tau) \tau^{l}\right) \\
& \quad=\hat{A}_{11}(\tau) \sum_{k \leq 4} \phi_{k}(\log \tau) \tau^{k}+\left(1+\hat{A}_{12}(\tau)\right) \sum_{k \leq 3} \psi_{k}(\log \tau) \tau^{k} \\
& \sum_{n \leq 2} \sum_{l=n+1}^{3} \frac{1}{(l-n) !} \frac{d^{l-n}}{d \tau^{l-n}}\left(\psi_{l}(\log \tau) \tau^{l}\right) \\
& \quad=\hat{A}_{21}(\tau) \sum_{k \leq 4} \phi_{k}(\log \tau) \tau^{k}+\hat{A}_{22}(\tau) \sum_{k \leq 3} \psi_{k}(\log \tau) \tau^{k}
\end{aligned}
$$

We solve equations (25) and (26) in the class of formal series in $\tau$ with coefficients that are polynomials in $\log \tau$. Putting $u=\log \tau$ allows us to write, for any smooth function $f$,

$$
\tau \frac{d f(\log \tau)}{d \tau}=f^{\prime}(u)
$$

We have to show that (25) and (26) can be satisfied at every order in $\tau^{n}$ for $n \leq 3$. Identifying terms of degree $n-1$ in $\tau$ in (25) and terms of degree $n-2$ in $\tau$ in (26), we get

$$
\begin{aligned}
& \sum_{\ell=n}^{4} \frac{1}{\tau^{n-1}(\ell-n+1) !} \frac{d^{\ell-n+1}}{d \tau^{\ell-n+1}}\left(\phi_{\ell}(\log \tau) \tau^{\ell}\right) \\
& \quad=\left(\hat{A}_{11}(\tau) \sum_{\ell \leq 4} \phi_{\ell}(\log \tau) \tau^{\ell}+\left(1+\hat{A}_{12}(\tau)\right) \sum_{\ell \leq 3} \psi_{\ell}(\log \tau) \tau^{\ell}\right) \|_{n-1} \\
& \sum_{\ell=n-1}^{3} \frac{1}{\tau^{n-2}(\ell-n+2) !} \frac{d^{\ell-n+2}}{d \tau^{\ell-n+2}}\left(\psi_{\ell}(\log \tau) \tau^{\ell}\right) \\
& \quad=\left(\hat{A}_{21}(\tau) \sum_{\ell \leq 4} \phi_{\ell}(\log \tau) \tau^{\ell}+\hat{A}_{22}(\tau) \sum_{\ell \leq 3} \psi_{\ell}(\log \tau) \tau^{\ell}\right) \|_{n-2}
\end{aligned}
$$


We show that, for all integers $n \leq 4$, there are polynomial functions $\phi_{n}, \psi_{n-1}$ such that (27) and (28) are satisfied. We show this property by induction on $n$. Substituting $n=4$ into (27) and (28) leads to $\psi_{3}=4 \phi_{4}$. A solution of this equation is given by

$$
\phi_{4}=1, \quad \psi_{3}=4
$$

Then the general solution can be obtained from this one by multiplication by a constant. We continue by induction. Assume that there exists an integer $n \leq 4$ such that, for all $n+1 \leq k \leq 4$, both $\phi_{k}$ and $\psi_{k-1}$ are polynomial in $u=\log \tau$. We show now that $\phi_{n}$ and $\psi_{n-1}$ are also polynomial in $u$. The upper bound on their degree is to be checked later. Observe that

$$
\mathcal{R}_{n, 3}:=-\psi_{n-1}+\left(\hat{A}_{12}(\tau) \sum_{k \leq 3} \psi_{k} \tau^{k}\right) \|_{n-1}=\sum_{\ell=n-4}^{-2} A_{12, \ell} \psi_{n-1-\ell}
$$

and

$$
\mathcal{R}_{n, 2}:=\left(\hat{A}_{11}(\tau) \sum_{k \leq 4} \phi_{k} \tau^{k}\right) \|_{n-1}=\sum_{\ell=n-5}^{-2} A_{11, \ell} \phi_{n-1-\ell} .
$$

Furthermore, since $A_{21,-2}=12$, we have

$$
\mathcal{S}_{n-1,2}:=-12 \psi_{n}+\left(\hat{A}_{21}(\tau) \sum_{k \leq 3} \phi_{k} \tau^{k}\right) \|_{n-2}=\sum_{\ell=n-6}^{-2} A_{21, \ell} \phi_{n-2-\ell} .
$$

Moreover, we have

$$
\mathcal{S}_{n-1,3}:=\left(\hat{A}_{21}(\tau) \sum_{k \leq 3} \psi_{k} \tau^{k}\right) \|_{n-2}=\sum_{\ell=n-5}^{-2} A_{22, \ell} \psi_{n-2-\ell} .
$$

Also,

$$
\begin{aligned}
\sum_{\ell=n}^{4} \frac{1}{\tau^{n-1}(\ell-n+1) !} \frac{d^{\ell-n+1}}{d \tau^{\ell-n+1}}\left(\phi_{\ell}(\log \tau) \tau^{\ell}\right) & =n \phi_{n}+\phi_{n}^{\prime}-\mathcal{R}_{n, 1}, \\
\sum_{\ell=n-1}^{3} \frac{1}{\tau^{n+1}(\ell-n+2) !} \frac{d^{\ell-n+2}}{d \tau^{\ell-n+2}}\left(\psi_{\ell}(\log \tau) \tau^{\ell}\right) & =(n-1) \psi_{n-1}+\psi_{n-1}^{\prime}-\mathcal{S}_{n-1,1},
\end{aligned}
$$

where

$$
\begin{aligned}
\mathcal{R}_{n, 1} & =-\frac{1}{\tau^{n-1}} \sum_{l=n+1}^{4} \frac{d^{l-n+1}\left(\phi_{l} \tau^{l}\right)}{d \tau^{l-n+1}}, \\
\mathcal{S}_{n-1,1} & =-\frac{1}{\tau^{n-2}} \sum_{l=n}^{3} \frac{d^{l-n+2}\left(\psi_{l} \tau^{l}\right)}{d \tau^{l-n+2}} .
\end{aligned}
$$

Then equations (27) and (28) take the form

$$
\left(\begin{array}{c}
\phi_{n} \\
\psi_{n-1}
\end{array}\right)^{\prime}(u)=\left(\begin{array}{cc}
-n & 1 \\
12 & -n+1
\end{array}\right)\left(\begin{array}{c}
\phi_{n} \\
\psi_{n-1}
\end{array}\right)(u)+\left(\begin{array}{c}
\mathcal{R}_{n} \\
\mathcal{S}_{n-1}
\end{array}\right)(u),
$$


where

$$
\begin{aligned}
\mathcal{R}_{n} & =\mathcal{R}_{n, 1}+\mathcal{R}_{n, 2}+\mathcal{R}_{n, 3}, \\
\mathcal{S}_{n-1} & =\mathcal{S}_{n-1,1}+\mathcal{S}_{n-1,2}+\mathcal{S}_{n-1,3}
\end{aligned}
$$

are polynomial functions in $u$. Equation (35) is a linear non-homogeneous equation in $\Phi_{n}=\left(\phi_{n}, \psi_{n-1}\right)$ with constant coefficients and a polynomial right-hand side. It can be rewritten in vector form as

$$
\dot{\boldsymbol{\Phi}}_{n}=\mathrm{M}_{n} \boldsymbol{\Phi}_{n}+\mathcal{R}_{n}
$$

where

$$
\mathcal{R}_{n}=\left(\begin{array}{c}
\mathcal{R}_{n} \\
\mathcal{S}_{n-1}
\end{array}\right), \quad \mathbf{M}_{n}=\left(\begin{array}{cc}
-n & 1 \\
12 & -n+1
\end{array}\right) .
$$

Matrix $\mathrm{M}_{n}$ has eigenvalues $\{-3-n, 4-n\}$ and can be diagonalized by a linear substitution defined by the matrix

$$
\mathrm{H}=\left(\begin{array}{cc}
-1 & 1 \\
3 & 4
\end{array}\right)
$$

The latter matrix does not depend on $n$. We write $\Phi_{n}=\mathrm{H} \tilde{\boldsymbol{\Phi}}_{n}$ and after substitution into (36) we get

$$
\dot{\tilde{\boldsymbol{\Phi}}}_{n}=\mathrm{D}_{n} \tilde{\boldsymbol{\Phi}}_{n}+\mathrm{H}^{-1} \mathcal{R}_{n},
$$

where

$$
\mathrm{D}_{n}=\left(\begin{array}{cc}
-3-n & 0 \\
0 & 4-n
\end{array}\right) .
$$

After that the equation can be easily integrated. The general solution of (37) has the form

$$
\begin{gathered}
\tilde{\phi}_{n}(u)=\bar{R}_{n}(u)+K_{1, n} e^{-(3+n) u}, \\
\tilde{\psi}_{n-1}(u)=\bar{S}_{n-1}(u)+K_{2, n} e^{(4-n) u},
\end{gathered}
$$

where both $K_{1, n}$ and $K_{2, n}$ are constant, and $\bar{R}_{n}(u)$ and $\bar{S}_{n-1}(u)$ are polynomial functions. Therefore, the choice $K_{1, n}=K_{2, n}=0$ leads to a polynomial solution of (35). If $n \notin$ $\{-3,4\}$, this solution is unique. The coefficients of $\Phi$ can be restored using

$$
\left(\begin{array}{c}
\phi_{n} \\
\psi_{n-1}
\end{array}\right)=\mathrm{H}\left(\begin{array}{c}
\tilde{\phi}_{n} \\
\tilde{\psi}_{n-1}
\end{array}\right) \text {. }
$$

In the case where $n \neq-3$, the degree of these polynomials is not higher than $\max \left\{\mathrm{d}^{0}\left(\mathcal{R}_{n}\right), \mathrm{d}^{0}\left(\mathcal{S}_{n-1}\right)\right\}$. In the case where $n=-3$, the degree of these polynomials is not higher than $\max \left\{\mathrm{d}^{0}\left(\mathcal{R}_{n}\right), \mathrm{d}^{0}\left(\mathcal{S}_{n-1}\right)\right\}+1$. This shows that the $\phi_{k}$ and $\psi_{k-1}, k \leq 4$, are polynomial in $u$ and are defined uniquely up to a free constant, which appears at the orders $n=4$ and $n=-3$.

We now show by induction on $n$ that their degree is not higher than $4-n$ in the case $n>-3$ and not higher than $5-n$ when $n \leq-3$. Assume first that there exists an integer $n \geq-3$ such that, for all integer $n<k \leq 4$,

$$
\max \left\{\mathrm{d}^{0}\left(\phi_{k}\right), \mathrm{d}^{0}\left(\psi_{k-1}\right)\right\} \leq 4-k .
$$

\section{CAMBRIDGE JOURNALS}


With (33), (30), (29), (34), (31) and (32), this implies that

$$
\begin{gathered}
\mathrm{d}^{0}\left(\mathcal{R}_{n, 1}\right) \leq 3-n, \quad \mathrm{~d}^{0}\left(\mathcal{R}_{n, 2}\right) \leq 3-n, \quad \mathrm{~d}^{0}\left(\mathcal{R}_{n, 3}\right) \leq 2-n, \\
\mathrm{~d}^{0}\left(\mathcal{S}_{n-1,1}\right) \leq 3-n, \quad \mathrm{~d}^{0}\left(\mathcal{S}_{n-1,2}\right) \leq 4-n, \quad \mathrm{~d}^{0}\left(\mathcal{S}_{n-1,3}\right) \leq 3-n,
\end{gathered}
$$

and therefore that

$$
\max \left\{\mathrm{d}^{0}\left(\mathcal{R}_{n}\right), \mathrm{d}^{0}\left(\mathcal{S}_{n-1}\right)\right\} \leq 4-n .
$$

Since the polynomial solution in (38) is of degree not higher than $\max \left\{\mathrm{d}^{0}\left(\phi_{k}\right), \mathrm{d}^{0}\left(\psi_{k-1}\right)\right\}$, (40) is valid for all $k$ such that $-3<k \leq 4$. If $n=-3$, the polynomial solutions of (38) are of degree not higher than $5-n$. Assume now that there exists an integer $n \leq-3$ such that, for all integer $n<k$,

$$
\max \left\{\mathrm{d}^{0}\left(\phi_{k}\right), \mathrm{d}^{0}\left(\psi_{k-1}\right)\right\} \leq 5-k,
$$

and let us show that (41) is still valid for $k=n$. With (33), (30), (29), (34), (31) and (32), it follows that

$$
\begin{gathered}
\mathrm{d}^{0}\left(\mathcal{R}_{n, 1}\right) \leq 4-n, \quad \mathrm{~d}^{0}\left(\mathcal{R}_{n, 2}\right) \leq 4-n, \quad \mathrm{~d}^{0}\left(\mathcal{R}_{n, 3}\right) \leq 3-n, \\
\mathrm{~d}^{0}\left(\mathcal{S}_{n-1,1}\right) \leq 4-n, \quad \mathrm{~d}^{0}\left(\mathcal{S}_{n-1,2}\right) \leq 5-n, \quad \mathrm{~d}^{0}\left(\mathcal{S}_{n-1,3}\right) \leq 5-n,
\end{gathered}
$$

and therefore the polynomial solutions of (38) are of degree not higher than $5-n$ and (41) is still valid for all integer $n \leq-3$.

In the case of a generic matrix $\hat{A}$ the upper bounds on the order of the polynomials $\phi_{k}$ and $\psi_{k-1}$ are sharp and cannot be further improved. However, if $\hat{A}(\tau)=F^{\prime}(\hat{\boldsymbol{\Gamma}}(\tau))$ it can be checked that the leading order in (37) cancels at $n=-3$, and the order of $\left(\phi_{k}^{+}, \psi_{k-1}^{+}\right)$ does not exceed $4-k$ not only for $4 \geq k>-3$ but for all $k \leq 4$.

\section{Inverse linear operators}

In this paper most of the analyticity results are proved by reducing the corresponding problem to a contraction mapping in a suitably chosen Banach space. Typically the reduction procedure requires the study of a linear operator and its inverse. In this section we provide corresponding details on the theory of linear finite-difference operators. We note that the properties of an operator depend both on its form and on its domain.

Let $r>r_{0}>0,0<\delta<\pi / 4$. The norms of all operators involved in this section depend on those parameters. We fix $\delta$ arbitrarily and keep it constant throughout our proofs; in contrast the parameter $r_{0}$ will be later chosen to ensure certain upper bounds.

In what follows we will consider functions analytic in one of the following subsets of $\mathbb{C}$ :

$$
\begin{aligned}
& \mathcal{D}_{+}(r)=\{\tau \in \mathbb{C}|| \arg (\tau-r) \mid<\pi-\delta\}, \\
& \mathcal{D}_{-}(r)=-\mathcal{D}_{+}(r)=\left\{\tau \in \mathbb{C} \mid-\tau \in \mathcal{D}_{+}(r)\right\}, \\
& \mathcal{D}_{1}(r)=\mathcal{D}_{-}(r) \cap \mathcal{D}_{+}(r) \cap\{\tau \mid \operatorname{Im} \tau<-r\} .
\end{aligned}
$$

We note that if $r>r_{0}$ then $\mathcal{D}_{+}(r) \subset \mathcal{D}_{+}\left(r_{0}\right)$.

Let $\mathcal{D} \subset \mathbb{C}$ and $p>0$. We say that $\mathbf{f}: \mathcal{D} \rightarrow \mathbb{C}^{2}$ belongs to $\mathfrak{X}_{p}(\mathcal{D})$, if $\mathbf{f}=\left(f_{x}, f_{y}\right)$ is analytic inside $\mathcal{D}$, continuous in its closure and has a finite norm:

$$
\|\mathbf{f}\|_{p}=\sup _{\tau \in \mathcal{D}}\left(\left|\tau^{p} f_{x}\right|+\left|\tau^{p+1} f_{y}\right|\right) .
$$


We also write

$$
\mathbf{f}(\tau)=\left(\tau^{-p} f_{1}(\tau), \tau^{-p-1} f_{2}(\tau)\right) \quad \text { then }\|\mathbf{f}\|_{p}=\sup _{\tau \in \mathcal{D}}\left(\left|f_{1}(\tau)\right|+\left|f_{2}(\tau)\right|\right) .
$$

Let $\mathrm{A}$ be a matrix-valued function,

$$
\mathrm{A}(\tau)=\left(\begin{array}{ll}
1 & 1 \\
0 & 1
\end{array}\right)+\left(\begin{array}{ll}
A_{11}(\tau) & A_{12}(\tau) \\
A_{21}(\tau) & A_{22}(\tau)
\end{array}\right) .
$$

We study the finite-difference operator, which acts by the formula

$$
\mathrm{L}(\boldsymbol{\Phi})(\tau)=\boldsymbol{\Phi}(\tau+1)-\mathrm{A}(\tau) \boldsymbol{\Phi}(\tau) .
$$

We will consider this operator on various different classes of functions. In the following we assume that $\mathrm{A}(\tau)$ satisfies the following assumptions.

[A1] $A_{k l} \in \mathfrak{X}_{2}\left(\mathcal{D}_{+}\left(r_{0}\right)\right)$ for $k, l \in\{1,2\}$.

[A2] There are formal series of the form

$$
\hat{A}_{k l}(\tau)=\sum_{m \leq-2} A_{k l, m}(\log \tau) \tau^{m},
$$

where $A_{k l, m}(z)$ are polynomials of order $-(m+2)$ for all $m \leq-2$, such that

$$
A_{k l}(\tau) \simeq \hat{A}_{k l}(\tau) \quad \text { as } \tau \rightarrow \infty, \tau \in \mathcal{D}_{+}\left(r_{0}\right),
$$

where $\simeq$ means that the function $A_{k l}$ is asymptotic to the series $\hat{A}_{k l}$. We do not assume convergence of the series $\hat{A}_{k l}(\tau)$.

[A3] $A_{2} 1,-2=12$.

THEOREM 4.1. Let $p>4$ and $r_{0}>0$. Suppose that A satisfies assumptions [A1]-[A3]. Then the linear operator $\mathrm{L}: \mathfrak{X}_{p}\left(\mathcal{D}_{+}\left(r_{0}\right)\right) \rightarrow \mathfrak{X}_{p}\left(\mathcal{D}_{+}\left(r_{0}\right)\right)$ has a trivial kernel. Moreover, there is $r_{0}^{\prime} \geq r_{0}$ such that for every $r \geq r_{0}^{\prime}$ there exists a unique continuous linear operator $\mathrm{L}^{-1}: \mathfrak{X}_{p+1}\left(\mathcal{D}_{+}(r)\right) \rightarrow \mathfrak{X}_{p}\left(\mathcal{D}_{+}(r)\right)$ such that $\mathrm{LL}^{-1}=\mathrm{Id}$. The norm of $\mathrm{L}^{-1}$ is bounded by a constant $C_{1}$ independent of $r$.

We will also consider the operator $\mathrm{L}$ restricted to functions analytic in $\mathcal{D}_{1}(r)$. Unlike the set $\mathcal{D}_{+}(r)$, the set $\mathcal{D}_{1}(r)$ is not invariant under the translation $\mathrm{T}^{-1}: \tau \rightarrow \tau-1$. Therefore, if $\Phi$ is analytic in $\mathcal{D}_{1}(r)$, then $\mathrm{L}(\boldsymbol{\Phi})$ is defined on a smaller domain

$$
\mathcal{D}_{1}^{\prime}(r)=\mathcal{D}_{1}(r) \cap \mathrm{T}^{-1}\left(\mathcal{D}_{1}(r)\right)
$$

due to the presence of the term $\boldsymbol{\Phi}(\tau+1)$ in the definition of $\mathrm{L}$.

We say that a matrix-valued solution of the equation $\mathrm{LU}=0$ is a fundamental solution if $\mathrm{U}$ is analytic in $\mathcal{D}_{+}\left(r_{0}\right)$ and continuous in its closure, its Wronskian $\omega(\tau):=\operatorname{det} \mathrm{U}(\tau)$ does not vanish in $\mathcal{D}_{+}\left(r_{0}\right)$ and $\omega(\tau) \rightarrow 1$ as $\tau \rightarrow \infty$ inside the domain.

THEOREM 4.2. Suppose that A satisfies the assumptions of Theorem 4.1 and $r>r_{0}^{\prime}$. Then the linear operator $\mathrm{L}: \mathfrak{X}_{p}\left(\mathcal{D}_{1}(r)\right) \rightarrow \mathfrak{X}_{p}\left(\mathcal{D}_{1}^{\prime}(r)\right)$ has a kernel consisting of the functions of the form

$$
\mathrm{U}(\tau) \mathbf{c}(\tau)
$$

where $\mathrm{U}(\tau)$ is the fundamental matrix solution of the homogeneous equation $\mathrm{L}(\mathrm{U})=0$ and $\boldsymbol{c}(\tau+1)=\boldsymbol{c}(\tau)$ and $\boldsymbol{c}(\tau) \rightarrow 0$ as $\tau \rightarrow \infty$ in $\mathcal{D}_{1}(r)$. The operator $\mathrm{L}$ has a bounded right inverse $\mathrm{L}^{-1}: \mathfrak{X}_{p+3}\left(\mathcal{D}_{1}^{\prime}(r)\right) \rightarrow \mathfrak{X}_{p}\left(\mathcal{D}_{1}(r)\right)$. The norm of $\mathrm{L}^{-1}$ is bounded by a constant $C_{2}$ independent of $r$. 
On this class of functions, the kernel of $\mathrm{L}$ is not trivial and the right inverse operator $\mathrm{L}^{-1}$ is not unique.

We also stress a subtle point in the previous two theorems related to the domains of the right inverse operator $\mathrm{L}^{-1}$ : its domain does not totally cover the range of $\mathrm{L}$.

The next sections contain a proof of Theorems 4.1 and 4.2. First we prove that Theorem 4.1 is valid for a specially chosen operator $L_{0}$. Then we use perturbation theory as every operator $\mathrm{L}$ can be considered as a small perturbation of the operator $\mathrm{L}_{0}$. The role of the perturbation parameter is played by the parameter $r_{0}^{\prime}$, which is involved in the definition of the domains. A large part of the proof is dedicated to a construction of a fundamental solution $\mathrm{U}$ of the equation $\mathrm{L}(\mathrm{U})=0$.

4.1. Fundamental solutions for $\mathrm{L}_{0}$. We consider the finite-difference operator defined by

$$
\mathrm{L}_{0}(\boldsymbol{\Phi})(\tau)=\boldsymbol{\Phi}(\tau+1)-\mathrm{A}_{0}(\tau) \Phi(\tau)
$$

where

$$
\mathrm{A}_{0}=\left(\begin{array}{cc}
1+12 / \tau^{2} & 1 \\
12 / \tau^{2} & 1
\end{array}\right)
$$

The equation

$$
\mathrm{L}_{0}(\boldsymbol{\Phi})=0
$$

admits two special solutions,

$$
\boldsymbol{\Phi}_{0}^{+}=\left(\begin{array}{c}
\phi_{0}^{+} \\
\psi_{0}^{+}
\end{array}\right), \quad \boldsymbol{\Phi}_{0}^{-}=\left(\begin{array}{l}
\phi_{0}^{-} \\
\psi_{0}^{-}
\end{array}\right),
$$

which we describe here. The first solution is polynomial,

$$
\left\{\begin{array}{l}
\phi_{0}^{+}(\tau)=\tau^{4}+\frac{1}{5} \tau^{2}, \\
\psi_{0}^{+}(\tau)=4 \tau^{3}+6 \tau^{2}+\frac{22}{5} \tau-\frac{6}{5},
\end{array}\right.
$$

and can be easily verified by substituting it directly into the equation rewritten in coordinate form:

$$
\left\{\begin{array}{l}
\phi_{0}(\tau+1)=\phi_{0}(\tau)+\psi_{0}(\tau+1), \\
\psi_{0}(\tau+1)=\left(12 / \tau^{2}\right) \phi_{0}(\tau)+\psi_{0}(\tau) .
\end{array}\right.
$$

The second solution is found by variation of constants. We note that $\operatorname{det}\left(\mathrm{A}_{0}\right)=1$. Then we can require the second solution to satisfy the normalization condition

$$
w_{0}(\tau):=\operatorname{det}\left|\begin{array}{ll}
\phi_{0}^{+} & \phi_{0}^{-} \\
\psi_{0}^{+} & \psi_{0}^{-}
\end{array}\right|(\tau)=1 .
$$

We find the second solution $\left(\phi_{0}^{-}, \psi_{0}^{-}\right)$eliminating $\psi_{0}^{+}$and $\psi_{0}^{-}$using the first line of (50), which can be rewritten in the form

$$
\begin{aligned}
& \psi_{0}^{+}(\tau)=\phi_{0}^{+}(\tau)-\phi_{0}^{+}(\tau-1), \\
& \psi_{0}^{-}(\tau)=\phi_{0}^{-}(\tau)-\phi_{0}^{-}(\tau-1),
\end{aligned}
$$


and then substituting $\phi_{0}^{-}(\tau)=k(\tau) \phi_{0}^{+}(\tau)$ into the normalizing condition (51). We get

$$
\begin{gathered}
\phi_{0}^{+}(\tau)\left[k(\tau) \phi_{0}^{+}(\tau)-k(\tau-1) \phi_{0}^{+}(\tau-1)\right] \\
-\left[\phi_{0}^{+}(\tau)-\phi_{0}^{+}(\tau-1)\right] k(\tau) \phi_{0}^{+}(\tau)=1,
\end{gathered}
$$

and therefore

$$
\kappa(\tau)-\kappa(\tau-1)=\frac{1}{\phi_{0}^{+}(\tau) \phi_{0}^{+}(\tau-1)} .
$$

This equation has an analytic solution in $\mathbb{C} \backslash \mathbb{R}_{\text {- defined by }}$

$$
\kappa(\tau)=-\sum_{n=1}^{\infty} \frac{1}{\phi_{0}^{+}(\tau+n) \phi_{0}^{+}(\tau+n-1)} .
$$

The series obviously converges for all $\tau$ from the domain. We restore the solution of equation (50) by

$$
\left\{\begin{array}{l}
\phi_{0}^{-}(\tau)=-\sum_{n=1}^{\infty} \frac{\phi_{0}^{+}(\tau)}{\phi_{0}^{+}(\tau+n) \phi_{0}^{+}(\tau+n-1)} \\
\psi_{0}^{-}(\tau)=\phi_{0}^{-}(\tau)-\phi_{0}^{-}(\tau-1)
\end{array}\right.
$$

We note that

$$
\phi_{0}^{-}(\tau)=-\frac{1}{7} \tau^{-3}+O\left(\tau^{-4}\right) \quad \text { and } \quad \psi_{0}^{-}(\tau)=\frac{3}{7} \tau^{-4}+O\left(\tau^{-5}\right)
$$

in any $D_{+}(r)$ with $r \geq 1$. This estimate can be easily derived by bounding the infinite sum by the corresponding integrals $[\mathbf{9}, \mathbf{1 3}]$.

For later convenience, we write

$$
\mathrm{U}_{0}=\left(-\frac{1}{84} \boldsymbol{\Phi}_{0}^{+},-84 \boldsymbol{\Phi}_{0}^{-}\right)
$$

to denote the fundamental system of solutions for the equation $\mathrm{L}_{0} \mathrm{U}_{0}=0$. The constant factor was chosen to ensure that the second column of $U_{0}$ starts with $12 \tau^{-3}$, similar to the formal series $\hat{\boldsymbol{\Gamma}}^{\prime}(\tau)$.

4.2. Inverting $\mathrm{L}_{0}$. We now come to the construction of the inverse of the operator $\mathrm{L}_{0}$. Note that the procedure used in this section does not use the specific form of the matrix $\mathrm{A}_{0}$, and we will use the same method to invert other operators on $\mathfrak{X}_{p}\left(\mathcal{D}_{+}(r)\right)$ and, after a proper modification, on $\mathfrak{X}_{p}\left(\mathcal{D}_{1}(r)\right)$ as well.

Let us consider the equation

$$
\mathrm{L}_{0}(\boldsymbol{\xi})=\mathbf{f},
$$

where $\mathbf{f} \in \mathfrak{X}_{p}\left(D_{+}\left(r_{0}\right)\right)$ with $p>4$. Taking into account the definition of $\mathrm{L}_{0}$, we rewrite this equation in the form

$$
\boldsymbol{\xi}(\tau+1)=\mathrm{A}_{0}(\tau) \boldsymbol{\xi}(\tau)+\mathbf{f}(\tau) .
$$

Let $\mathrm{U}_{0}$ be the fundamental solution constructed in $\S 4.1$. We look for a solution of (56) in the form $\xi(\tau)=\mathrm{U}_{0}(\tau) \boldsymbol{\eta}(\tau)$. After a substitution into (56) this leads to

$$
\begin{aligned}
\mathrm{U}_{0}(\tau+1) \eta(\tau+1) & =\mathrm{A}_{0}(\tau) \mathrm{U}_{0}(\tau) \boldsymbol{\eta}(\tau)+\mathbf{f}(\tau) \\
& =\mathrm{U}_{0}(\tau+1) \boldsymbol{\eta}(\tau)+\mathbf{f}(\tau) .
\end{aligned}
$$


The matrix $\mathrm{U}_{0}$ is invertible, therefore

$$
\boldsymbol{\eta}(\tau+1)-\boldsymbol{\eta}(\tau)=\mathrm{U}_{0}^{-1}(\tau+1) \mathbf{f}(\tau) .
$$

This is an elementary finite-difference equation, which admits the solution

$$
\eta(\tau)=-\sum_{k=0}^{\infty} \mathrm{U}_{0}^{-1}(\tau+1+k) \mathbf{f}(\tau+k) .
$$

The series converges uniformly in $\mathcal{D}_{+}\left(r_{0}\right)$ and, consequently, defines an analytic function. Therefore

$$
\xi(\tau)=-\mathrm{U}_{0}(\tau) \sum_{k=0}^{\infty} \mathrm{U}_{0}^{-1}(\tau+1+k) \mathbf{f}(\tau+k)
$$

is analytic in $\mathcal{D}_{+}\left(r_{0}\right)$.

Let us prove that the norm of $\mathrm{L}_{0}^{-1}: \mathfrak{X}_{p}\left(\mathcal{D}_{+}(r)\right) \rightarrow \mathfrak{X}_{p-1}\left(\mathcal{D}_{+}(r)\right)$ is bounded by a constant independent of $r>r_{0}$. Write

$$
\mathrm{U}_{0}(\tau)=\left(\begin{array}{ll}
U_{0,11}(\tau) \tau^{4} & U_{0,12}(\tau) \tau^{-3} \\
U_{0,21}(\tau) \tau^{3} & U_{0,22}(\tau) \tau^{-4}
\end{array}\right)
$$

and let

$$
U_{0, \max }:=\max _{1 \leq i, j \leq 2} \sup _{\tau \in \mathcal{D}_{+}\left(r_{0}\right)}\left\{\left|U_{0, i j}(\tau)\right|\right\}<\infty .
$$

We have $\operatorname{det} \mathrm{U}_{0}(\tau) \equiv 1$, therefore

$$
\mathrm{U}_{0}^{-1}(\tau)=\left(\begin{array}{cc}
U_{0,22}(\tau) \tau^{-4} & -U_{0,12}(\tau) \tau^{-3} \\
-U_{0,21}(\tau) \tau^{3} & U_{0,11}(\tau) \tau^{4}
\end{array}\right),
$$

and we have an upper bound for the inverse matrix as well as for the $\mathrm{U}_{0}$. Then

$$
\mathrm{U}_{0}(\tau) \mathrm{U}_{0}^{-1}(\tau+1+k) \mathbf{f}(\tau+k)=\left(\begin{array}{ll}
V_{11}(\tau, \tau+k) & V_{12}(\tau, \tau+k) \\
V_{21}(\tau, \tau+k) & V_{22}(\tau, \tau+k)
\end{array}\right)\left(\begin{array}{l}
f_{1}(\tau+k) \\
f_{2}(\tau+k)
\end{array}\right),
$$

where

$$
\begin{aligned}
V_{11}(\tau, \tau+k)= & (\tau+k)^{-p}\left[U_{0,11}(\tau) U_{0,22}(\tau+k) \tau^{4}(\tau+k)^{-4}\right. \\
& \left.-U_{0,12}(\tau) U_{0,21}(\tau+k) \tau^{-3}(\tau+k)^{3}\right], \\
V_{12}(\tau, \tau+k)= & (\tau+k)^{-p-1}\left[U_{0,12}(\tau) U_{0,11}(\tau+k) \tau^{-3}(\tau+k)^{4}\right. \\
& \left.-U_{0,11}(\tau) U_{0,12}(\tau+k) \tau^{4}(\tau+k)^{-3}\right], \\
V_{21}(\tau, \tau+k)= & (\tau+k)^{-p}\left[U_{0,21}(\tau) U_{0,22}(\tau+k) \tau^{3}(\tau+k)^{-4}\right. \\
& \left.-U_{0,22}(\tau) U_{0,21}(\tau+k) \tau^{-4}(\tau+k)^{3}\right], \\
V_{22}(\tau, \tau+k)= & (\tau+k)^{-p-1}\left[U_{0,22}(\tau) U_{0,11}(\tau+k) \tau^{-4}(\tau+k)^{4}\right. \\
& \left.-U_{0,21}(\tau) U_{0,12}(\tau+k) \tau^{3}(\tau+k)^{-3}\right] .
\end{aligned}
$$

These coefficients admit the following upper bounds for all $\tau \in \mathcal{D}_{+}\left(r_{0}\right)$, and all $k \geq 0$ :

$$
\begin{aligned}
& \left|V_{11}(\tau, \tau+k)\right| \leq U_{0, \text { max }}^{2}\left(|\tau|^{4}|\tau+k|^{-p-4}+|\tau|^{-3}|\tau+k|^{-p+3}\right), \\
& \left|V_{12}(\tau, \tau+k)\right| \leq U_{0, \text { max }}^{2}\left(|\tau|^{-3}|\tau+k|^{-p+3}+|\tau|^{4}|\tau+k|^{-p-4}\right), \\
& \left|V_{21}(\tau, \tau+k)\right| \leq U_{0, \text { max }}^{2}\left(|\tau|^{3}|\tau+k|^{-p-4}+|\tau|^{-4}|\tau+k|^{-p+3}\right), \\
& \left|V_{22}(\tau, \tau+k)\right| \leq U_{0, \text { max }}^{2}\left(|\tau|^{-4}|\tau+k|^{-p+3}-|\tau|^{3}|\tau+k|^{-p-4}\right) .
\end{aligned}
$$


Since, for each $p>0$, there exists $K_{p, r_{0}}>0$ such that, for all $\tau \in \mathcal{D}_{+}\left(r_{0}\right)$,

$$
\sum_{k=0}^{\infty} \frac{1}{|\tau+k|^{p+1}} \leq \frac{K_{p, r_{0}}}{|\tau|^{p}}
$$

it follows from the comparison theorem that the series for $\boldsymbol{\xi}$ converges absolutely and uniformly in every domain $\mathcal{D}_{+}(r)$ with $r>r_{0}$, and

$$
\left\|\mathrm{L}_{0}^{-1}(\mathbf{f})\right\|_{p-1} \leq U_{0, \max }^{2} 2\left(K_{p+4, r_{0}}+K_{p-3, r_{0}}\right)\|\mathbf{f}\|_{p-1} .
$$

Theorem 4.1 is proved for the operator $\mathrm{L}_{0}$ and $r_{0}^{\prime} \geq r_{0}$ for any fixed $r_{0}>0$.

4.3. Analytic fundamental solutions. Let $\hat{U}$ be a formal fundamental solution of the equation $\mathrm{L} \hat{\mathrm{U}}=0$ defined using solutions provided by Lemma 3.1. It is not difficult to check that the coefficients can be chosen in such a way that the formal series $\operatorname{det} U(\tau)$ starts with the constant term equal to 1 .

LEMMA 4.1. Let a matrix-valued function A satisfy the assumptions of Theorem 4.1 and $\hat{\mathrm{U}}(\tau)$ be a formal fundamental solution. Then there is $r_{0}^{\prime}>r_{0}$ such that the equation $\mathrm{LU}=0$ has a unique fundamental solution in $\mathcal{D}_{+}\left(r_{0}^{\prime}\right)$, which is asymptotic to $\hat{\mathrm{U}}$ as $\tau \rightarrow \infty$ inside the domain.

Proof. Let $\mathrm{B}(\tau)=\mathrm{A}(\tau)-\mathrm{A}_{0}(\tau)$, where $\mathrm{A}_{0}$ is defined by (47). Then

$$
\mathrm{B}(\tau)=\left(\begin{array}{cc}
\tau^{-2} B_{11}(\tau) & \tau^{-2} B_{12}(\tau) \\
\tau^{-3} \log \tau B_{21}(\tau) & \tau^{-2} B_{22}(\tau)
\end{array}\right),
$$

where $B_{11}, B_{12}, B_{21}, B_{22}$ are bounded in $\mathcal{D}_{+}\left(r_{0}\right)$ due to assumption [A2]. Take a negative integer $N<-4$ and consider a partial sum $\hat{\mathrm{U}}_{N}$ of the formal solution $\hat{\mathrm{U}}$,

$$
\mathrm{U}_{N}(\tau)=\left(\begin{array}{ll}
\phi_{N}^{+}(\tau) & \phi_{N}^{-}(\tau) \\
\psi_{N}^{+}(\tau) & \psi_{N}^{-}(\tau)
\end{array}\right)
$$

which includes all terms up to order $N$. More precisely,

$$
\left\{\begin{array}{l}
\phi_{N}^{+}(\tau)=\sum_{k=N}^{4} \phi_{k}^{+}(\log \tau) \tau^{k} \\
\psi_{N}^{+}(\tau)=\sum_{k=N-1}^{3} \psi_{k}^{+}(\log \tau) \tau^{k}
\end{array}\right.
$$

and

$$
\left\{\begin{array}{l}
\phi_{N}^{-}(\tau)=\sum_{k=N}^{-3} \phi_{k}^{-}(\log \tau) \tau^{k}, \\
\psi_{N}^{-}(\tau)=\sum_{k=N-1}^{-4} \psi_{k}^{-}(\log \tau) \tau^{k} .
\end{array}\right.
$$

The matrix-valued function $\mathrm{U}_{N}$ is an approximate solution of the equation $\mathrm{LU}=0$ in the sense that

$$
\mathcal{H}_{N}:=\mathrm{L}\left(\mathrm{U}_{N}\right)
$$


belongs to $\mathfrak{X}_{p+1}\left(\mathcal{D}_{+}\left(r_{0}\right)\right)$ for every $p \in(-N,-N+1)$, i.e. each of the two columns of the matrix belongs to the space.

We look for the fundamental solution in the form

$$
\mathrm{U}(\tau)=\mathrm{U}_{N}(\tau)+\mathcal{V}(\tau)
$$

where $\mathcal{V}$ should belong to $\mathfrak{X}_{p}\left(\mathcal{D}_{+}\left(r_{0}\right)\right)$. We substitute this formula and $\mathrm{A}(\tau)=\mathrm{A}_{0}(\tau)+$ $\mathrm{B}(\tau)$ into the equation

$$
\mathrm{U}(\tau+1)=\mathrm{A}(\tau) \mathrm{U}(\tau),
$$

and rewrite the result in the following form:

$$
\mathcal{V}(\tau+1)-\mathrm{A}_{0}(\tau) \mathcal{V}(\tau)=\mathrm{B}(\tau) \mathcal{V}(\tau)+\mathcal{H}_{N}(\tau)
$$

The operator $\mathrm{L}_{0}(\mathcal{V}(\tau))=\mathcal{V}(\tau+1)-\mathrm{A}_{0}(\tau) \mathcal{V}(\tau)$ is invertible in $\mathfrak{X}_{p}\left(\mathcal{D}_{+}\left(r_{0}\right)\right)$. Therefore in this space the last equation is equivalent to

$$
\mathcal{V}(\tau)=\mathrm{L}_{0}^{-1}(\mathrm{~B}(\tau) \mathcal{V}(\tau))+\mathrm{L}_{0}^{-1}\left(\mathcal{H}_{N}(\tau)\right)
$$

In order to prove the existence and uniqueness of a solution, it suffices to check that there is a constant $r_{0}^{\prime}>r_{0}$ such that the map

$$
\mathcal{W}(\tau) \mapsto \mathrm{L}_{0}^{-1}(\mathrm{~B}(\tau) \mathcal{W}(\tau))+\mathrm{L}_{0}^{-1}\left(\mathcal{H}_{N}(\tau)\right)
$$

is a contraction of $\mathfrak{X}_{p}\left(\mathcal{D}_{+}\left(r_{0}^{\prime}\right)\right)$. This latter will be guaranteed if, for all $\mathbf{g} \in \mathcal{X}_{p}\left(\mathcal{D}_{+}\left(r_{0}^{\prime}\right)\right)$,

$$
\left\|\mathrm{L}_{0}^{-1}(\mathrm{Bg})\right\|_{p}<\frac{1}{2}\|\mathbf{g}\|_{p} .
$$

The norm of the operator

$$
\mathrm{L}_{0}: \mathfrak{X}_{p+1}\left(\mathcal{D}_{+}\left(r_{0}^{\prime}\right)\right) \rightarrow \mathfrak{X}_{p}\left(\mathcal{D}_{+}\left(r_{0}^{\prime}\right)\right)
$$

is bounded by a constant, which is independent of $r_{0}^{\prime}>r_{0}$ (but depends on $r_{0}$ ). We write

$$
\mathbf{g}(\tau)=\left(\tau^{-p} g_{1}(\tau), \tau^{-p-1} g_{2}(\tau)\right),
$$

where both $g_{1}$ and $g_{2}$ are bounded and $\|\mathbf{g}\|_{p}=\sup _{\tau \in \mathcal{D}_{+}\left(r_{0}^{\prime}\right)}\left(\left|g_{1}(\tau)\right|+\left|g_{2}(\tau)\right|\right)$. The map $\mathbf{g}(\tau) \mapsto \mathrm{B}(\tau) \mathbf{g}(\tau)$ is linear. Taking into account (61) we get

$$
\begin{aligned}
\|\mathrm{B}(\tau) \mathbf{g}(\tau)\|_{p+1} \leq \sup _{\tau \in \mathcal{D}_{+}\left(r_{0}^{\prime}\right)}\left(|\tau|^{-1}\left|B_{11}(\tau) g_{1}(\tau)\right|+|\tau|^{-2}\left|B_{12}(\tau) g_{2}(\tau)\right|,\right. & \\
& \left.|\tau|^{-1}|\log \tau|\left|B_{21}(\tau) g_{1}(\tau)\right|+|\tau|^{-1}\left|B_{22}(\tau) g_{2}(\tau)\right|\right) .
\end{aligned}
$$

The function $\left|\tau^{-1 / 2} \log \tau\right|$ is bounded in $\mathcal{D}_{+}\left(r_{0}\right)$ and there is a constant $K_{r_{0}}$ such that

$$
\|\mathrm{Bg}\|_{p+1} \leq \frac{K_{r_{0}}}{\sqrt{r_{0}^{\prime}}}\|\mathbf{g}\|_{p} .
$$

Therefore

$$
\left\|\mathrm{L}_{0}^{-1}(\mathrm{Bg})\right\|_{p}<\frac{\left\|L_{0}^{-1}\right\| K_{r_{0}}}{\sqrt{r_{0}^{\prime}}}\|\mathbf{g}\|_{p},
$$

and the condition (66) is satisfied for every $r_{0}^{\prime}>4 \sqrt{\left\|L_{0}^{-1}\right\| K_{r_{0}}}$. Then the contraction mapping theorem implies that there is a unique solution of $(65)$ in $\mathfrak{X}_{p}\left(\mathcal{D}_{+}\left(r_{0}^{\prime}\right)\right)$.

This proves that, for every $N<-4$, the difference $\mathrm{U}(\tau)-\mathrm{U}_{N}(\tau)$ belongs to $\mathfrak{X}_{p}\left(\mathcal{D}_{+}\left(r_{0}^{\prime}\right)\right)$ for $p \in(-N,-N+1)$, which is equivalent to $\mathrm{U}(\tau) \simeq \mathrm{U}_{N}(\tau)$ as $\tau \rightarrow \infty$ in $\mathcal{D}_{+}\left(r_{0}^{\prime}\right)$. 
4.4. Kernel of L. In this short section we prove the statements about the kernel of the operator $\mathrm{L}$ from Theorems 4.1 and 4.2.

Let $\boldsymbol{\varphi} \in \mathfrak{X}_{p}\left(\mathcal{D}_{+}(r)\right)$ satisfy the homogeneous equation $\mathrm{L} \boldsymbol{\varphi}=0$. Let $\mathrm{U}$ be the fundamental solution of the equation defined in the previous section. Note that for every $\tau \in \mathcal{D}_{+}(r)$ we have det $\mathrm{U}(\tau) \neq 0$. Consider the auxiliary function $\mathbf{c}(\tau)=\mathrm{U}^{-1}(\tau) \varphi(\tau)$. We immediately get

$$
\begin{aligned}
\mathbf{c}(\tau+1) & =\mathrm{U}^{-1}(\tau+1) \varphi(\tau+1)=(\mathrm{A}(\tau) \mathrm{U}(\tau))^{-1} \mathrm{~A}(\tau) \varphi(\tau) \\
& =\mathrm{U}(\tau)^{-1} \boldsymbol{\varphi}(\tau)=\mathbf{c}(\tau) .
\end{aligned}
$$

We conclude that $\mathbf{c}(\tau)=\mathbf{c}(\tau+k)$ for every $k \in \mathbb{N}$. At the same time $\tau+k \in \mathcal{D}_{+}(r)$ and $\mathrm{U}(\tau)=O\left(\tau^{4}\right)$. Then $\varphi \in \mathfrak{X}_{p}\left(\mathcal{D}_{+}(r)\right)$ with $p>4$ implies that

$$
c(\tau)=\lim _{k \rightarrow \infty} \mathbf{c}(\tau+k)=\lim _{k \rightarrow \infty} \mathrm{U}^{-1}(\tau+k) \boldsymbol{\varphi}(\tau+k)=0 .
$$

Therefore, $c(\tau) \equiv 0$, and the kernel of $\mathrm{L}: \mathfrak{X}_{p}\left(\mathcal{D}_{+}(r)\right) \rightarrow \mathfrak{X}_{p}\left(\mathcal{D}_{+}(r)\right)$ is trivial.

This finishes the proof of the first part of Theorem 4.1.

On the other hand, if $\varphi \in \mathfrak{X}_{p}\left(\mathcal{D}_{1}(r)\right)$ the chain of equalities (69) is still valid. Moreover, $\mathrm{U}(\tau)=O\left(\tau^{4}\right)$ and $p>4$ imply that $\lim _{\tau \rightarrow \infty} \mathbf{c}(\tau)=0$, where the limit is taken for $\tau \in \mathcal{D}_{1}(r)$. In this case the kernel of $\mathrm{L}$ consists of functions of the form $\mathrm{U}(\tau) \mathbf{c}(\tau)$, i.e. every solution of the homogeneous equation is a linear combination of two basic solutions with periodic coefficients.

This finishes the proof of the first part of Theorem 4.2.

Note that in this case we cannot repeat the argument to show that $\mathbf{c}$ is zero because for every $\tau \in \mathcal{D}_{1}(r)$ only a finite number of points $\tau+k$ also belong to $\mathcal{D}_{1}(r)$. So we cannot take the limit $k \rightarrow \infty$.

4.5. Inverting $\mathrm{L}$. In this section we show that $\mathrm{L}^{-1}$ is bounded by a constant independent of $r>r_{0}^{\prime}$.

Proof of Theorem 4.1. (Second part) Similarly to $\$ 4.2$ we check that

$$
\xi=\mathrm{L}^{-1} \mathbf{f}(\tau)=-\mathrm{U}(\tau) \sum_{k=0}^{\infty} \mathrm{U}^{-1}(\tau+1+k) \mathbf{f}(\tau+k)
$$

satisfies the equation $\mathrm{L} \boldsymbol{\xi}=\mathbf{f}$ provided the series converges.

Let $p>4$ and $r>r_{0}^{\prime}$. For any $\mathbf{f} \in \mathcal{X}_{p}$, we write $\mathbf{f}=\left(f_{1}(\tau) \tau^{-p}, f_{2}(\tau) \tau^{-p-1}\right)$, then

$$
\|\mathbf{f}\|_{p}=\sup _{\mathcal{D}_{+}(r)}\left(\left|f_{1}(\tau)\right|+\left|f_{2}(\tau)\right|\right)<\infty .
$$

Similarly, we introduce the functions $U_{k l}$ by

$$
\mathrm{U}(\tau)=\left(\begin{array}{ll}
U_{11}(\tau) \tau^{4} & U_{12}(\tau) \tau^{-3} \\
U_{21}(\tau) \tau^{3} & U_{22}(\tau) \tau^{-4}
\end{array}\right)
$$

There is a constant $U_{\max }>1$ such that

$$
\max _{1 \leq i, j \leq 2} \sup _{\tau \in \mathcal{D}_{+}\left(r_{0}\right)}\left\{\left|U_{i j}(\tau)\right|\right\}=U_{\max }, \quad \frac{1}{U_{\max }} \leq|\operatorname{det} \mathrm{U}(\tau)| \leq U_{\max } .
$$


Then we get an upper bound for the elements of the inverse matrix:

$$
\mathrm{U}^{-1}(\tau)=\frac{1}{\operatorname{det} \mathrm{U}(\tau)}\left(\begin{array}{cc}
U_{22}(\tau) \tau^{-4} & -U_{12}(\tau) \tau^{-3} \\
-U_{21}(\tau) \tau^{3} & U_{11}(\tau) \tau^{4}
\end{array}\right)=\left(\begin{array}{cc}
\tau^{-4} V_{11}(\tau) & \tau^{-3} V_{12}(\tau) \\
\tau^{3} V_{21}(\tau) & \tau^{4} V_{22}(\tau)
\end{array}\right)
$$

We obtain

$$
\max _{1 \leq i, j \leq 2} \sup _{\tau \in \mathcal{D}_{+}\left(r_{0}\right)}\left\{\left|V_{i j}(\tau)\right|\right\} \leq U_{\max }^{2}
$$

It follows that, for each $\tau \in \mathcal{D}_{+}(r)$, we have

$$
\mathrm{U}^{-1}(\tau+1) \mathbf{f}(\tau)=\left(\begin{array}{c}
\tau^{-4-p} V_{11}(\tau+1) f_{1}(\tau)+\tau^{-4-p} V_{12}(\tau+1) f_{2}(\tau) \\
\tau^{3-p} V_{21}(\tau+1) f_{1}(\tau)+\tau^{3-p} V_{22}(\tau+1) f_{2}(\tau)
\end{array}\right) .
$$

Combining together these upper bounds, (59) and (70), we obtain

$$
\|\boldsymbol{\xi}\|_{p-1} \leq 4 K_{p} U_{\max }^{3}\|\mathbf{f}\|_{p} .
$$

This implies that if $p>4$ and $r>r_{0}^{\prime}$ then the operator $\mathrm{L}^{-1}: \mathfrak{X}_{p}\left(\mathcal{D}_{+}(r)\right) \rightarrow \mathfrak{X}_{p-1}\left(\mathcal{D}_{+}(r)\right)$ defined by (70) is bounded by a constant that does not depend on $r$.

Theorem 4.1 is proved.

4.6. Elementary finite-difference equation. If convergent, each of the sums

$$
\eta(\tau)=-\sum_{j=0}^{\infty} g(\tau+j) \quad \text { and } \quad \eta(\tau)=\sum_{j=1}^{\infty} g(\tau-j)
$$

solves the equation

$$
\eta(\tau+1)-\eta(\tau)=g(\tau)
$$

Unfortunately, neither of them is well defined if $g$ is defined in $D_{1}(r)$ only. In order to overcome this difficulty, we use an analytical version of the partition of unity proposed by Lazutkin in [13]. We represent $g$ as a sum of two functions: one has an analytic continuation to the left of the domain and the other one to the right. The method is based on a partition of unity on the boundary of the domain in combination with Cauchy integrals used to construct analytic functions.

LeMma 4.2. Let $r \geq 2, \delta>0, \tan \delta \leq \frac{1}{2}, \alpha \geq 0$ and $q \geq 4$. If $g: D_{1}(r) \rightarrow \mathbb{C}$ is analytic, continuous in the closure of its domain and there is $M_{g}>0$ such that

$$
|g(\tau)| \leq \frac{M_{g}}{\left|\tau^{q} e^{i \alpha \tau}\right|} \quad \text { for all } \tau \in D_{1}(r)
$$

then the equation

$$
\eta(\tau+1)-\eta(\tau)=g(\tau)
$$

has an analytic solution in $D_{1}(r)$, continuous in the closure of the domain and

$$
|\eta(\tau)| \leq \frac{2 \pi}{r(\sin \delta)^{q-3}} \frac{M_{g}}{\left|\tau^{q-3} e^{i \alpha \tau}\right|} .
$$


Proof. The proof is a straightforward generalization of corresponding proofs from $[9,13]$. As the explicit upper bound for the solution of the elementary finite-difference equation (76) is important for the validity of our main theorems, we provide all the main details.

Consider the following two domains

$$
\begin{aligned}
& D_{2}^{-}(r)=\{\tau \in \mathbb{C} \mid \arg (\tau-r-1)<-\delta, \operatorname{Im} \tau<-r\}, \\
& D_{2}^{+}(r)=\{\tau \in \mathbb{C} \mid \arg (\tau+r)>-\pi+\delta, \operatorname{Im} \tau<-r\} .
\end{aligned}
$$

We note that $D_{1}(r)=D_{2}^{-}(r) \cap D_{2}^{+}(r)$. Using the Cauchy integral and a partition of unity on the boundary $\partial \mathrm{D}_{1}(r)$, we can prove the following lemma.

LEMMA 4.3. Let $r>2, \delta>0, \tan \delta<\frac{1}{2}$. Let $\tilde{g}$ be analytic in $D_{1}(r)$ and continuous in the closure of the domain. If there is a constant $\tilde{M}_{\tilde{g}}$ such that

$$
|\tilde{g}(\tau)| \leq \tilde{M}_{\tilde{g}}|\tau|^{-2}
$$

on its domain, then there are two functions $P_{+}(\tilde{g})$ and $P_{-}(\tilde{g})$ analytic in $D_{2}^{ \pm}(r)$ respectively, continuous in the closure of their domains and

$$
\left|P_{ \pm}(\tilde{g})(\tau)\right| \leq \frac{2}{r} \tilde{M}_{\tilde{g}}
$$

such that

$$
P_{+}(\tilde{g})(\tau)+P_{-}(\tilde{g})(\tau)=\tilde{g}(\tau)
$$

for all $\tau \in D_{1}(r)$.

We skip the proof of this lemma as it is almost identical to [9, Proposition 9.4].

Now we consider the weight function $\mu(\tau)=\tau^{q-2} e^{i \alpha \tau}$. The function $\tilde{g}=\mu g$ obviously satisfies Lemma 4.3, and $\tilde{M}_{\tilde{g}}=M_{g}$. A solution to the equation (76) is given by the formula

$$
\eta(\tau)=\sum_{j=1}^{\infty} \frac{P_{-}(\mu g)(\tau-j)}{\mu(\tau-j)}-\sum_{j=0}^{\infty} \frac{P_{+}(\mu g)(\tau+j)}{\mu(\tau+j)} .
$$

Indeed, it is not difficult to see that the series converge if $\tau \in D_{1}(r)$ and $p>3$. Moreover,

$$
\eta(\tau+1)-\eta(\tau)=\frac{P_{-}(\mu g)(\tau)}{\mu(\tau)}+\frac{P_{+}(\mu g)(\tau)}{\mu(\tau)}=\frac{P_{-}(\mu g)(\tau)+P_{+}(\mu g)(\tau)}{\mu(\tau)}=g(\tau) .
$$

This solution admits the following upper bound:

$$
|\eta(\tau)| \leq \frac{2 M_{g}}{r} \sum_{j=-\infty}^{+\infty} \frac{1}{|\tau+j|^{q-2}\left|e^{i \alpha \tau}\right|} \leq \frac{2 \pi M_{g}}{r} \frac{1}{|\operatorname{Im} \tau|^{q-3}\left|e^{i \alpha \tau}\right|},
$$

where the sum was bounded by an integral under the assumption $q \geq 4$. In $D_{1}(r)$ we have $|\operatorname{Im} \tau| \geq|\tau| \sin \delta$, which implies the desired estimate for the solution. 
4.7. End of the proof of Theorem 4.2. When $\mathbf{f}$ is defined in the domain $\mathcal{D}_{1}^{\prime}(r)$, the series (70) is not well defined and cannot be used to solve the equation

$$
\mathrm{L} \xi=\mathbf{f} \text {. }
$$

Instead, we reduce this equation to the elementary finite-difference equation studied in the previous section. Let $\mathrm{U}(\tau)$ be a fundamental solution of the homogeneous equation defined by Lemma 4.1. Then $\operatorname{det} \mathrm{U}(\tau) \neq 0$ for $\tau \in \mathcal{D}_{1}^{\prime}(r)$ and the matrix $\mathrm{U}(\tau)$ is invertible. Taking into account the definition of $\mathrm{L}$, we observe that $\boldsymbol{\xi}(\tau)=\mathrm{U}(\tau) \boldsymbol{\eta}(\tau)$ solves the equation provided that

$$
\eta(\tau-1)-\eta(\tau)=\mathrm{U}^{-1}(\tau+1) \mathbf{f}(\tau) .
$$

We apply Lemma 4.2 with $\alpha=0$ to solve this system. We use $q=p+4$ for the first equation of the system and $q=p-3$ for the second one. The inverse matrix has the form (72) and $\mathbf{f} \in \mathfrak{X}_{p}\left(\mathcal{D}^{\prime}{ }_{+}(r)\right)$, so we can check that we can take

$$
M_{\mathbf{g}}=U_{\max }^{2}\|\mathbf{f}\|_{p}
$$

Lemma 4.2 implies that there is a solution of the system (78)

$$
\boldsymbol{\eta}(\tau)=\left(\tau^{-p-1} \eta_{1}(\tau), \tau^{-p+6} \eta_{2}(\tau)\right)
$$

such that

$$
\begin{aligned}
\left|\eta_{1}(\tau)\right| & \leq \frac{2 \pi U_{\max }^{2}}{r(\sin \delta)^{p+1}}\|\mathbf{f}\|_{p}, \\
\left|\eta_{2}(\tau)\right| & \leq \frac{2 \pi U_{\max }^{2}}{r(\sin \delta)^{p-6}}\|\mathbf{f}\|_{p} .
\end{aligned}
$$

Then we use (71) to conclude that

$$
\|\boldsymbol{\xi}\|_{p-3}=\|\mathrm{U} \boldsymbol{\eta}\|_{p-3} \leq \frac{8 \pi U_{\max }^{3}}{r(\sin \delta)^{p-6}}\|\mathbf{f}\|_{p},
$$

which provides the desired uniform estimate for the solution of the equation. The map $\mathrm{L}^{-1}: \mathbf{f} \mapsto \xi$ is a right inverse to $\mathrm{L}$. Theorem 4.2 is proved.

\section{Proof of Theorem 2.1}

Take $n \in \mathbb{N}, n>4$. Let $\hat{\boldsymbol{\Gamma}}_{n}$ be a partial sum of the formal separatrix $\hat{\boldsymbol{\Gamma}}$, which includes terms up to order $O\left(\tau^{-n}\right)$ in its first component and up to $O\left(\tau^{-n-1}\right)$ in the second one. The function $\hat{\boldsymbol{\Gamma}}_{n}(\tau)$ converges to zero as $\tau$ goes to infinity, and consequently there is $r_{1}>0$ such that

$$
\boldsymbol{R}_{n}(\tau):=\hat{\boldsymbol{\Gamma}}_{n}(\tau+1)-F\left(\hat{\boldsymbol{\Gamma}}_{n}(\tau)\right)
$$

is well defined and belongs to the space $\mathfrak{X}_{n+1+\epsilon}\left(\mathcal{D}_{+}\left(r_{1}\right)\right)$ for every $\epsilon \in(0,1)$. Note that generically $\boldsymbol{R}_{n}$ does not belong to $\mathfrak{X}_{n+2}\left(\mathcal{D}_{+}\left(r_{1}\right)\right)$ due to the presence of logarithmic terms.

Let us look for the stable separatrix in the form

$$
\Gamma^{s}(\tau)=\hat{\boldsymbol{\Gamma}}_{n}(\tau)+\boldsymbol{\xi}(\tau)
$$


Substituting this into equation (6) we obtain

$$
\boldsymbol{\xi}(\tau+1)=F\left(\hat{\boldsymbol{\Gamma}}_{n}(\tau)+\boldsymbol{\xi}(\tau)\right)-F\left(\hat{\boldsymbol{\Gamma}}_{n}(\tau)\right)-\boldsymbol{R}_{n}(\tau) .
$$

It is convenient to rewrite this equation in the form

$$
\mathrm{L} \xi=\mathrm{Q}(\boldsymbol{\xi})-\boldsymbol{R}_{n},
$$

where we use the notation

$$
\begin{aligned}
\mathrm{Q}(\boldsymbol{\xi})= & F\left(\hat{\boldsymbol{\Gamma}}_{n}(\tau)+\boldsymbol{\xi}(\tau)\right)-F\left(\hat{\boldsymbol{\Gamma}}_{n}(\tau)\right)-F^{\prime}\left(\hat{\boldsymbol{\Gamma}}_{n}(\tau)\right) \boldsymbol{\xi} \\
& (\mathrm{L} \boldsymbol{\xi})(\tau)=\boldsymbol{\xi}(\tau+1)-F^{\prime}\left(\hat{\boldsymbol{\Gamma}}_{n}(\tau)\right) \boldsymbol{\xi}(\tau) .
\end{aligned}
$$

Theorem 4.1 implies that there is $r_{2}>r_{1}$ such that for every $r>r_{2}$ the operator L has a uniformly bounded right inverse. In $\mathfrak{X}_{n}\left(\mathcal{D}_{+}(r)\right)$, this equation is equivalent to the 'integral' equation

$$
\xi=\mathrm{L}^{-1} \mathrm{Q}(\xi)-\mathrm{L}^{-1} \boldsymbol{R}_{n},
$$

where the inverse operator $\mathrm{L}^{-1}$ is defined by Theorem 4.1 , and its norm is bounded by a constant $C_{1}$. Let

$$
\rho=2\left\|\boldsymbol{R}_{n}\right\|_{n+1} C_{1} .
$$

We note that if $r>r_{2}$ then $\mathfrak{X}_{n}\left(\mathcal{D}_{+}\left(r_{2}\right)\right) \subset \mathfrak{X}_{n}\left(\mathcal{D}_{+}(r)\right)$ and the norm of the embedding map equals 1 . Consequently,

$$
\left\|\mathrm{L}^{-1} \boldsymbol{R}_{n}\right\|_{n} \leq \rho / 2 .
$$

We show that there is $r_{3}>r_{2}$ such that the right-hand side of equation (80) is a contraction in a ball $B_{\rho} \subset \mathfrak{X}_{n}\left(\mathcal{D}_{+}\left(r_{3}\right)\right)$, which implies the existence of a unique fixed point inside this ball. We have to check two statements: $B_{\rho}$ is invariant, and the restriction of the nonlinear operator on this ball is a contraction.

Invariant ball. Taking into account (4) and rewriting the definition of $\mathrm{Q}(\boldsymbol{\xi})$ in coordinates, we obtain

$$
\mathrm{Q}(\boldsymbol{\xi})=\left(\begin{array}{l}
a\left(\hat{\boldsymbol{\Gamma}}_{n}(\tau)+\boldsymbol{\xi}(\tau)\right)-a\left(\hat{\boldsymbol{\Gamma}}_{n}(\tau)\right)-\nabla a \cdot \boldsymbol{\xi}(\tau) \\
b\left(\hat{\boldsymbol{\Gamma}}_{n}(\tau)+\boldsymbol{\xi}(\tau)\right)-b\left(\hat{\boldsymbol{\Gamma}}_{n}(\tau)\right)-\nabla b \cdot \boldsymbol{\xi}(\tau)
\end{array}\right),
$$

where $\nabla a$ and $\nabla b$ denote gradients of the functions $a$ and $b$ respectively. Consider the auxiliary functions

$$
\begin{aligned}
& \varphi_{1}(t)=a\left(\hat{\boldsymbol{\Gamma}}_{n}(\tau)+t \boldsymbol{\xi}(\tau)\right)-a\left(\hat{\boldsymbol{\Gamma}}_{n}(\tau)\right)-t \nabla a \cdot \boldsymbol{\xi}(\tau), \\
& \varphi_{2}(t)=b\left(\hat{\boldsymbol{\Gamma}}_{n}(\tau)+t \boldsymbol{\xi}(\tau)\right)-b\left(\hat{\boldsymbol{\Gamma}}_{n}(\tau)\right)-t \nabla b \cdot \boldsymbol{\xi}(\tau) .
\end{aligned}
$$

Obviously $\mathrm{Q}(\boldsymbol{\xi})=\left(\varphi_{1}(1), \varphi_{2}(1)\right)^{\mathrm{T}} . \quad$ Moreover, $\varphi_{1}(0)=\varphi_{1}^{\prime}(0)=\varphi_{2}(0)=\varphi_{2}^{\prime}(0)=0$. Integrating by parts we show that

$$
\varphi_{1}(1)=\int_{0}^{1}(1-t) \varphi_{1}^{\prime \prime}(t) d t \quad \text { and } \quad \varphi_{2}(1)=\int_{0}^{1}(1-t) \varphi_{2}^{\prime \prime}(t) d t
$$

Then there are constants $t_{1}, t_{2} \in[0,1]$ such that

$$
\varphi_{1}(1)=\left(1-t_{1}\right) \varphi_{1}^{\prime \prime}\left(t_{1}\right) \text { and } \varphi_{2}(1)=\left(1-t_{2}\right) \varphi_{2}^{\prime \prime}\left(t_{2}\right) .
$$


Consequently,

$$
\|\mathrm{Q}(\boldsymbol{\xi})\|_{n+1} \leq \sup _{\tau \in \mathcal{D}_{+}\left(r_{3}\right)}\left(\left|\tau^{n+1} \varphi_{1}^{\prime \prime}\left(t_{1}\right)\right|+\left|\tau^{n+2} \varphi_{2}^{\prime \prime}\left(t_{2}\right)\right|\right) .
$$

The second derivatives $\varphi_{1}^{\prime \prime}\left(t_{1}\right)$ and $\varphi_{2}^{\prime \prime}\left(t_{2}\right)$ can be easily written in terms of second partial derivatives of the functions $a$ and $b$ and the components of the vector $\xi$. The partial derivatives are bounded by the $C^{2}$-norm $\|F\|_{C^{2}}$ and we get

$$
\|\mathrm{Q}(\xi)\|_{n+1} \leq 8\|F\|_{C^{2}}\|\xi\|_{n}^{2} \sup _{\tau \in \mathcal{D}_{+}\left(r_{3}\right)}|\tau|^{n-2}=\frac{8\|F\|_{C^{2}}\|\boldsymbol{\xi}\|_{n}^{2}}{r_{3}^{n-2} \sin ^{n-2} \delta} .
$$

We conclude that, if $\xi \in B_{\rho}$ and

$$
r_{3}^{n-2}>\frac{16\|F\|_{C^{2}} C_{1} \rho^{2}}{\sin ^{n-2} \delta},
$$

then

$$
\left\|\mathrm{L}^{-1} \mathrm{Q}(\boldsymbol{\xi})-\mathrm{L}^{-1} \boldsymbol{R}_{n}\right\|_{n}<\rho,
$$

which implies that $B_{\rho}$ is invariant under the action of the map $\xi \mapsto \mathrm{L}^{-1} \mathrm{Q}(\boldsymbol{\xi})-\mathrm{L}^{-1} \boldsymbol{R}_{n}$.

Contraction. Let $\boldsymbol{\xi}, \boldsymbol{\eta} \in B_{\rho}$ and check that

$$
\left\|\mathrm{L}^{-1} \mathrm{Q}(\boldsymbol{\xi})-\mathrm{L}^{-1} \mathrm{Q}(\boldsymbol{\eta})\right\|_{n}<\frac{1}{8}\|\boldsymbol{\xi}-\boldsymbol{\eta}\|_{n} .
$$

Indeed, consider the straight line that connects points $\xi(\tau)$ and $\eta(\tau)$ :

$$
\boldsymbol{\theta}(t)=(1-t) \boldsymbol{\xi}(\tau)+t \boldsymbol{\eta}(\tau) .
$$

Obviously, $\boldsymbol{\theta}(0)=\boldsymbol{\xi}(\tau)$ and $\boldsymbol{\theta}(1)=\boldsymbol{\eta}(\tau)$. Similarly to the previous part of the proof, we define two auxiliary functions:

$$
\begin{aligned}
& \psi_{1}(t)=a\left(\hat{\boldsymbol{\Gamma}}_{n}(\tau)+\boldsymbol{\theta}(t)\right)-a\left(\hat{\boldsymbol{\Gamma}}_{n}(\tau)\right)-\nabla a\left(\hat{\boldsymbol{\Gamma}}_{n}(\tau)\right) \cdot \boldsymbol{\theta}(t), \\
& \psi_{2}(t)=b\left(\hat{\boldsymbol{\Gamma}}_{n}(\tau)+\boldsymbol{\theta}(t)\right)-b\left(\hat{\boldsymbol{\Gamma}}_{n}(\tau)\right)-\nabla b\left(\hat{\boldsymbol{\Gamma}}_{n}(\tau)\right) \cdot \boldsymbol{\theta}(t) .
\end{aligned}
$$

Obviously $\mathrm{Q}(\boldsymbol{\xi})=\left(\psi_{1}(0), \psi_{2}(0)\right)^{\mathrm{T}}$ and $\mathrm{Q}(\boldsymbol{\eta})=\left(\psi_{1}(1), \psi_{2}(1)\right)^{\mathrm{T}}$. The mean value theorem implies that there are constants $t_{1}, t_{2} \in[0,1]$ such that

$$
\psi_{1}(1)-\psi_{1}(0)=\psi_{1}^{\prime}\left(t_{1}\right) \quad \text { and } \quad \psi_{2}(1)-\psi_{2}(0)=\psi_{2}^{\prime}\left(t_{2}\right) .
$$

Then differentiating the definitions of $\psi_{j}$ we get

$$
\begin{aligned}
& \psi_{1}(1)-\psi_{1}(0)=\left[\nabla a\left(\hat{\boldsymbol{\Gamma}}_{n}(\tau)+\boldsymbol{\theta}\left(t_{1}\right)\right)-\nabla a\left(\hat{\boldsymbol{\Gamma}}_{n}(\tau)\right)\right] \cdot(\boldsymbol{\eta}(\tau)-\boldsymbol{\xi}(\tau)), \\
& \psi_{2}(1)-\psi_{2}(0)=\left[\nabla b\left(\hat{\boldsymbol{\Gamma}}_{n}(\tau)+\boldsymbol{\theta}\left(t_{2}\right)\right)-\nabla b\left(\hat{\boldsymbol{\Gamma}}_{n}(\tau)\right)\right] \cdot(\boldsymbol{\eta}(\tau)-\boldsymbol{\xi}(\tau)) .
\end{aligned}
$$

We note that $\boldsymbol{\theta}(t) \in B_{\rho}$ for all $t \in[0,1]$ and get upper bounds for the differences of the gradients in terms of $\|F\|_{C^{2}}$ and the length of the vector $\boldsymbol{\theta}(t)$, which directly imply the following upper bounds:

$$
\begin{aligned}
& \left|\psi_{1}(1)-\psi_{1}(0)\right| \leq\|F\|_{C^{2}} \rho|\tau|^{-2 n}\|\xi-\eta\|_{n}, \\
& \left|\psi_{2}(1)-\psi_{2}(0)\right| \leq\|F\|_{C^{2}} \rho|\tau|^{-2 n}\|\xi-\eta\|_{n} .
\end{aligned}
$$


Consequently, we have

$$
\begin{aligned}
\|\mathrm{Q}(\boldsymbol{\xi})-\mathrm{Q}(\boldsymbol{\eta})\|_{n+1} & =\sup _{\tau \in \mathcal{D}_{+}\left(r_{3}\right)}\left(\left|\tau^{n+1}\left(\psi_{1}(1)-\psi_{1}(0)\right)\right|+\left|\tau^{n+2}\left(\psi_{1}(1)-\psi_{1}(0)\right)\right|\right) \\
& \leq \sup _{\tau \in \mathcal{D}_{+}\left(r_{3}\right)}\left(2\|F\|_{C^{2}} \rho|\tau|^{2-n}\|\boldsymbol{\xi}-\eta\|_{n}\right) \\
& \leq \frac{2 \rho\|F\|_{C^{2}}}{r_{3}^{n-2} \sin ^{n-2} \delta}\|\boldsymbol{\xi}-\eta\|_{n} \leq \frac{\|\boldsymbol{\xi}-\eta\|_{n}}{8 C_{1}},
\end{aligned}
$$

where the last inequality is a corollary of (82). We apply the operator $\mathrm{L}^{-1}$ and immediately get (83) as $\left\|\mathrm{L}^{-1}\right\| \leq C_{1}$.

The contraction mapping theorem implies that equation (80) has a unique solution in the ball $B_{\rho}$ provided $r_{3}$ satisfies (82) and $\rho$ is defined by (81).

To finish the proof, we have to check that the separatrix solution $\Gamma^{s}(\tau)$ obtained in the proof with different $n$ is actually independent of the choice of $n$. Increasing $r_{3}$, if necessary, we can check that for any $n \geq 5$ the function $\Gamma^{s}(\tau)$ is sufficiently close to $\hat{\Gamma}_{5}(\tau)$ to ensure uniqueness due to the uniqueness of the fixed point proved for $n=5$.

Therefore we have proved the existence of a single analytic solution of equation (6) such that $\boldsymbol{\Gamma}^{s}(\tau) \simeq \hat{\boldsymbol{\Gamma}}(\tau)$ as $\tau \rightarrow \infty$ in $\mathcal{D}_{+}\left(r_{3}\right)$. Theorem 2.1 is proved.

\section{Proof of Theorem 2.2}

In this section we prove the exponential asymptotic for the difference between the stable and unstable separatrices. Let

$$
\xi_{*}(\tau)=\Gamma_{-}^{u}(\tau)-\Gamma^{s}(\tau) .
$$

It is obvious that $\xi \in \mathfrak{X}_{n}\left(\mathcal{D}_{1}(r)\right)$ for every $n \in \mathbb{N}$. Taking into account that $\Gamma_{-}^{u}(\tau)$ and $\Gamma^{s}(\tau)$ both satisfy (6), we obtain

$$
\xi_{*}(\tau+1)=F\left(\Gamma^{s}(\tau)+\xi_{*}(\tau)\right)-F\left(\Gamma^{s}(\tau)\right) .
$$

It is convenient to rewrite this equation in the form

$$
\mathrm{L} \xi_{*}=Q\left(\xi_{*}\right)
$$

where

$$
Q(\xi)=F\left(\Gamma^{s}(\tau)+\xi(\tau)\right)-F\left(\Gamma^{s}(\tau)\right)-F^{\prime}\left(\Gamma_{n}(\tau)\right) \xi(\tau)
$$

and

$$
(\mathrm{L} \xi)(\tau)=\boldsymbol{\xi}(\tau+1)-F^{\prime}\left(\Gamma^{s}(\tau)\right) \xi(\tau) .
$$

Choosing $n>4$ we use $\mathrm{L}^{-1}$ to denote the operator defined by Theorem 4.2. The function

$$
\xi_{0}:=\xi_{*}-\mathrm{L}^{-1} Q\left(\xi_{*}\right)
$$

satisfies the homogeneous equation $L \xi_{0}=0$. Let $U$ be the fundamental solution of the homogeneous equation $\mathrm{LU}=0$. By Theorem 4.2 there is a periodic vector-valued function $\mathbf{c}_{0}(\tau)$ such that

$$
\xi_{0}=\mathrm{Uc}_{0}
$$


We conclude that

$$
\xi_{*}=\mathrm{L}^{-1} Q\left(\boldsymbol{\xi}_{*}\right)+\mathrm{U} \mathbf{c}_{0} .
$$

This equation is very similar to (80). We can literally repeat the arguments of the previous sections, taking into account that the domain of $\mathrm{L}^{-1}$ is slightly different from the one used before.

It is convenient to choose $\rho=2\left\|\boldsymbol{\xi}_{*}\right\|$. Then we conclude that $\boldsymbol{\xi} \mapsto \mathrm{L}^{-1} Q(\boldsymbol{\xi})+\mathrm{Uc}_{0}$ is a contraction of the ball $\mathcal{B}_{\rho} \subset \mathfrak{X}_{n}\left(\mathcal{D}_{1}(r)\right)$ centred at the origin provided $r$ is sufficiently large, $r>r_{4}$, where

$$
r_{4}^{n-4}>\frac{16\|F\|_{C^{2}} C_{1} \rho^{2}}{\sin ^{n-4} \delta} .
$$

It follows that the sequence $\xi_{m}$ defined by

$$
\boldsymbol{\xi}_{m+1}=\mathrm{L}^{-1} Q\left(\boldsymbol{\xi}_{m}\right)+\mathrm{Uc}_{0}, \quad m \geq 0,
$$

converges to the unique fixed point in $B_{\rho}$. By uniqueness $\boldsymbol{\xi}_{*}=\lim _{m \rightarrow \infty} \boldsymbol{\xi}_{m}$.

Now we prove by induction that the sequence $\xi_{m}$ admits an exponentially small upper bound. Let

$$
\xi_{m}(\tau)=\left(\tau^{4} e^{2 \pi i \tau} \xi_{1, m}(\tau), \tau^{3} e^{2 \pi i \tau} \xi_{2, m}(\tau)\right)
$$

We will prove that there is a constant $C_{*}>0$ such that

$$
C_{m}:=\max \left\{\left|\xi_{1, m}(\tau)\right|,\left|\xi_{2, m}(\tau)\right|\right\}<C_{*},
$$

for all $m \geq 0$. To this purpose we construct another right inverse operator $\mathrm{L}^{-1}$.

If $\mathrm{L}_{1}^{-1}$ is another right inverse of $\mathrm{L}$ we can rewrite (84) in the form

$$
\boldsymbol{\xi}_{m+1}=\mathrm{L}_{1}^{-1} Q\left(\boldsymbol{\xi}_{m}\right)+\mathrm{Uc}_{m}+\mathrm{Uc}_{0},
$$

where

$$
\mathrm{U} \mathbf{c}_{m}=\mathrm{L}^{-1} Q\left(\xi_{m}\right)-\mathrm{L}_{1}^{-1} Q\left(\xi_{m}\right)
$$

is a solution of the homogeneous equation. We will construct $\mathrm{L}_{1}^{-1}$ using a procedure similar to the one of $\$ 4.7$. Unlike the case of $\mathrm{L}^{-1}$ we let $\alpha=4 \pi-\epsilon$ with a fixed small $\epsilon>0$. Let us check that $Q\left(\xi_{m}\right)$ is in the domain of $\mathrm{L}_{1}^{-1}$ and get an upper bound.

Let us get an upper bound for $Q(\xi)$. Taking into account that the map $F$ has the form (4), we get

$$
Q(\xi)=\left(\begin{array}{l}
a\left(\boldsymbol{\Gamma}^{s}+\xi\right)-a\left(\boldsymbol{\Gamma}^{s}\right)-\nabla a\left(\boldsymbol{\Gamma}^{s}\right) \cdot \xi \\
b\left(\boldsymbol{\Gamma}^{s}+\xi\right)-b\left(\boldsymbol{\Gamma}^{s}\right)-\nabla b\left(\boldsymbol{\Gamma}^{s}\right) \cdot \xi
\end{array}\right) .
$$

Both components of $Q\left(\xi_{m}\right)$ are bounded from above by

$$
2\|F\|_{C^{2}} C_{m}^{2}|\tau|^{8}\left|e^{-4 \pi i \tau}\right|
$$

Then taking into account (72) we conclude that the components of

$$
\mathbf{g}_{m}(\tau)=\mathrm{U}^{-1}(\tau+1) Q\left(\boldsymbol{\xi}_{m}(\tau)\right)
$$

are bounded from above by

$$
4 U_{\max }^{2}\|F\|_{C^{2}} C_{m}^{2}|\tau|^{4}\left|e^{-4 \pi i \tau}\right| \quad \text { and } \quad 4 U_{\max }^{2}\|F\|_{C^{2}} C_{m}^{2}|\tau|^{12}\left|e^{-4 \pi i \tau}\right|,
$$


respectively. We use Lemma 4.2 with $\alpha=4 \pi-\epsilon$ and $q=7$ or $q=8$ to get solutions of the equations:

$$
\eta_{1, m}(\tau+1)-\eta_{1}(\tau)=g_{1, m}(\tau) \quad \text { and } \quad \eta_{2, m}(\tau+1)-\eta_{2}(\tau)=g_{2, m}(\tau) .
$$

Let

$$
M_{\mathbf{g}_{m}}=4 U_{\max }^{2}\|F\|_{C^{2}} C_{m}^{2} \sup _{\mathcal{D}_{1}(r)}\left|\tau^{20} e^{-i \epsilon \tau}\right|,
$$

then

$$
\left|\eta_{1, m}(\tau)\right| \leq \frac{2 \pi M_{\mathbf{g}_{m}}}{r(\sin \delta)^{4}}\left|\tau^{-4} e^{-i \alpha \tau}\right| \quad \text { and } \quad\left|\eta_{2, m}(\tau)\right| \leq \frac{2 \pi M_{\mathbf{g}_{m}}}{r(\sin \delta)^{5}}\left|\tau^{-3} e^{-i \alpha \tau}\right| .
$$

Finally we take into account equation (71) and obtain that the components of the operator $L_{1}^{-1}\left(\mathbf{g}_{m}\right)=\mathrm{U}(\tau) \boldsymbol{\eta}_{m}(\tau)$ are both bounded by

$$
\frac{4 \pi U_{\max } M_{\mathbf{g}_{m}}}{r(\sin \delta)^{5}}\left|e^{-i \alpha \tau}\right| \text {. }
$$

Let

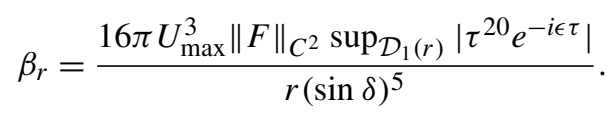

In this way we have established that the components of $\mathrm{L}_{1}^{-1} Q\left(\xi_{m}\right)$ are bounded by

$$
\beta_{r} C_{m}^{2}\left|e^{-(4 \pi-\epsilon) i \tau}\right| .
$$

Now we estimate $\mathbf{c}_{m}$. Let $\Delta_{\alpha, q}^{-1}$ denote the operator defined by Lemma 4.2. Then (86) implies that

$$
\mathbf{c}_{m}=\Delta_{0, n}^{-1} \mathbf{g}_{m}-\Delta_{\alpha, q}^{-1} \mathbf{g}_{m} .
$$

We have already derived the upper bound for the second term. In order to estimate the first one let

$$
\tilde{M}_{\mathbf{g}_{m}}=4 U_{\max }^{2}\|F\|_{C^{2}} C_{m}^{2} \sup _{\mathcal{D}_{1}(r)}\left|\tau^{n+12} e^{-4 \pi i \tau}\right|
$$

then

$$
\left|\tilde{\eta}_{1, m}(\tau)\right| \leq \frac{2 \pi M_{\mathbf{g}_{m}}}{r(\sin \delta)^{n-3}}\left|\tau^{-n+3}\right| \quad \text { and } \quad\left|\tilde{\eta}_{2, m}(\tau)\right| \leq \frac{2 \pi M_{\mathbf{g}_{m}}}{r(\sin \delta)^{n-3}}\left|\tau^{-n+3}\right| .
$$

Let

$$
\begin{aligned}
\alpha_{r}= & \frac{8 \pi U_{\max }^{2}\|F\|_{C^{2}} \sup _{\mathcal{D}_{1}(r)}\left|\tau^{n+12} e^{-4 \pi i \tau}\right|}{r^{n-2}(\sin \delta)^{n-3}} \\
& +\frac{8 \pi U_{\max }^{2}\|F\|_{C^{2} \sup _{\mathcal{D}_{1}(r)}\left|\tau^{20} e^{-i \epsilon \tau}\right|}}{r^{4}(\sin \delta)^{5}} e^{-(4 \pi-\varepsilon) r} .
\end{aligned}
$$

On the line $\operatorname{Im} \tau=r$ the components of the function $\mathbf{c}_{m}$ admit the following bound:

$$
\left|\mathbf{c}_{m}(\tau)\right| \leq \alpha_{r} C_{m}^{2}, \quad \operatorname{Im} \tau=r
$$

and $\lim _{r \rightarrow+\infty} \alpha_{r}=0$. The function $\mathbf{c}_{m}$ is periodic with period 1 and converges to 0 as $\operatorname{Im} \tau \rightarrow-\infty$, consequently

$$
\left|\mathbf{c}_{m}(\tau)\right| \leq \alpha_{r} C_{m}^{2} e^{2 \pi(r-\operatorname{Im} \tau)}, \quad \operatorname{Im} \tau \leq r .
$$


Using these estimates and (85) we conclude that

$$
C_{m+1} \leq C_{1}+\left(2 U_{\max } \alpha_{r} e^{2 \pi r}+\beta_{r} e^{-(2 \pi-\epsilon) r}\right) C_{m}^{2} .
$$

This implies that $C_{m}$ are uniformly bounded by $C_{*}=2 C_{1}$ provided that

$$
C_{1}\left(2 U_{\max } \alpha_{r} e^{2 \pi r}+\beta_{r} e^{-(2 \pi-\epsilon) r}\right)<\frac{1}{4} .
$$

Taking into account the definitions of $\alpha_{r}$ and $\beta_{r}$, it is easy to see that there is $r_{5}>0$ such that this condition is fulfilled for all $r>r_{5}$.

This implies an exponential upper bound on $\xi_{*}$. In order to finish the proof of the theorem, let

$$
\mathrm{U} \mathbf{c}_{*}=\xi_{*}-\mathrm{L}_{1}^{-1} Q\left(\xi_{*}\right) .
$$

This function satisfies the homogeneous equation $\mathrm{LU}=0$ and Theorem 4.2 implies that $\mathbf{c}_{*}$ is a decreasing periodic function. Denoting its first Fourier coefficient by $\boldsymbol{\Theta}^{-}$and taking into account that $U \asymp \hat{U}$ we obtain the first asymptotic expansion of the theorem.

The proof of the second asymptotic expansion follows the same arguments applied to functions in $\mathcal{D}_{1}^{+}(r)$.

Acknowledgement. This work is partially supported by the EPSRC grant EP/C000595/1.

\section{REFERENCES}

[1] A. Algaba, E. Freire, E. Gamero and C. García. Quasi-homogeneous normal forms. J. Comput. Appl. Math. 150(1) (2003), 193-216.

[2] A. Baider and J. Sanders. Further reduction of the Takens-Bogdanov normal form. J. Differential Equations 99(2) (1992), 205-244.

[3] R. I. Bogdanov. Versal deformations of a singular point on the plane in the case of zero eigenvalues. Funct. Anal. Appl. 9(2) (1975), 144-145.

[4] H. W. Broer, R. Roussarie and C. Simó. Invariant circles in the Bogdanov-Takens bifurcation for diffeomorphisms. Ergod. Th. \& Dynam. Sys. 16(6) (1996), 1147-1172.

[5] G. Chen, D. Wang and X. Wang. Unique normal forms for nilpotent planar vector fields. Internat. J. Bifur. Chaos Appl. Sci. Engrg. 12(10) (2002), 2159-2174.

[6] F. Dumortier, P. R. Rodrigues and R. Roussarie. Germs of Diffeomorphisms in the Plane (Lecture Notes in Mathematics, 902). Springer, Berlin, 1981, p. 197.

[7] J. Écalle. Nature du groupe des ordres d'itération complexes d'une transformation holomorphe au voisinage d'un point fixe de multiplicateur 1. C. R. Acad. Sci. Paris Sér. A-B 276 (1973), A261-A263 (in French).

[8] V. G. Gelfreich. Conjugation to a shift and splitting of separatrices. Appl. Math. 24(2) (1996), 127-140.

[9] V. G. Gelfreich. A proof of the exponentially small transversality of the separatrices for the standard map. Comm. Math. Phys. 201 (1999), 155-216.

[10] V. G. Gelfreich. Splitting of a small separatrix loop near the saddle-center bifurcation in area-preserving maps. Phys. D 136 (2000), 266-279.

[11] V. G. Gelfreich and D. Sauzin. Borel summation and the splitting of separatrices for the Hénon map. Ann. Inst. Fourier (Grenoble) 51(2) (2001), 513-567.

[12] V. Gelfreich. Chaotic zone in the Bogdanov-Takens bifurcation for diffeomorphisms. Analysis and Applications, ISAAC 2001. Eds. H. Begehr, R. Gilbert and M.-W. Wong. Kluwer Academic, Dordrecht, 2003, pp. 187-197. 
[13] V. F. Lazutkin. An analytic integral along the separatrix of the semistandard map: existence and an exponential estimate for the distance between the stable and unstable separatrices. Algebra $i$ Analiz 4(4) (1992), 110-142 (Engl. Transl. St. Petersburg Math. J. 4(4), (1993) 721-748).

[14] F. Takens. Forced oscillations and bifurcations. Applications of Global Analysis. I (Symp., Utrecht State University, Utrecht, 1973), Comm. Math. Inst. Rijksuniv. Utrecht 3 (1974), 1-59.

[15] S. M. Voronin. Analytic classification of germs of conformal mappings $(C, 0) \rightarrow(C, 0)$. Funktsional. Anal. i Prilozhen. 15(1) (1981), 1-17; 96 (in Russian).

\section{СамвRIDGE JUURNALS}

